\title{
Seismic wave attenuation and dispersion resulting from wave-induced flow in porous rocks - A review
}

\author{
Tobias M. Müller ${ }^{1}$, Boris Gurevich², and Maxim Lebedev ${ }^{3}$
}

\begin{abstract}
One major cause of elastic wave attenuation in heterogeneous porous media is wave-induced flow of the pore fluid between heterogeneities of various scales. It is believed that for frequencies below $1 \mathrm{kHz}$, the most important cause is the wave-induced flow between mesoscopic inhomogeneities, which are large compared with the typical individual pore size but small compared to the wavelength. Various laboratory experiments in some natural porous materials provide evidence for the presence of centimeter-scale mesoscopic heterogeneities. Laboratory and field measurements of seismic attenuation in fluid-saturated rocks provide indications of the role of the wave-induced flow. Signatures of wave-induced flow include the frequency and saturation dependence of P-wave attenuation and its associated velocity dispersion, frequency-dependent shear-wave splitting, and attenuation anisotropy. During the last four decades, numerous models for attenuation and velocity dispersion from wave-induced flow have been developed with varying degrees of rigor and complexity. These models can be categorized roughly into three groups ac-
\end{abstract}

cording to their underlying theoretical framework. The first group of models is based on Biot's theory of poroelasticity. The second group is based on elastodynamic theory where local fluid flow is incorporated through an additional hydrodynamic equation. Another group of models is derived using the theory of viscoelasticity. Though all models predict attenuation and velocity dispersion typical for a relaxation process, there exist differences that can be related to the type of disorder (periodic, random, space dimension) and to the way the local flow is incorporated. The differences manifest themselves in different asymptotic scaling laws for attenuation and in different expressions for characteristic frequencies. In recent years, some theoretical models of wave-induced fluid flow have been validated numerically, using finite-difference, finite-element, and reflectivity algorithms applied to Biot's equations of poroelasticity. Application of theoretical models to real seismic data requires further studies using broadband laboratory and field measurements of attenuation and dispersion for different rocks as well as development of more robust methods for estimating dissipation attributes from field data.

\section{INTRODUCTION}

Seismic waves in earth materials are subject to attenuation and dispersion in a broad range of frequencies and scales from free oscillations of the entire earth to ultrasound in small rock samples (Aki and Richards, 1980). Attenuation refers to the exponential decay of wave amplitude with distance; dispersion is a variation of propagation velocity with frequency. Attenuation and dispersion can be caused by a variety of physical phenomena that can be divided broadly into elastic processes, where the total energy of the wavefield is conserved (scattering attenuation, geometric dispersion), and inelastic dissipation, where wave energy is converted into heat. Of particular interest to exploration geophysics is inelastic attenuation and dispersion of body waves (P-and S-waves) resulting from the presence of fluids in the pore space of rocks. It is believed that an understanding of fluid-related dissipation in hydrocarbon reservoir rocks, combined with improved measurements of attenuation and/or dispersion from recorded seismic data, may be used in the future to estimate hydraulic properties of these rocks. Dissipation-related seismic attributes are already employed in seismic interpretation and reservoir characterization, but so far their use has been mostly empirical and qualitative. Theoretical models of frequency-dependent attenuation and dispersion may help develop quantitative attributes, which can be calibrated using well logs and laboratory measurements.

It is commonly accepted that the presence of fluids in the pore

Manuscript received by the Editor 20 January 2010; revised manuscript received 29 April 2010; published online 14 September 2010.

${ }^{1}$ CSIRO Earth Science and Resource Engineering, Perth, Australia. E-mail: tobias.mueller@ csiro.au.

${ }_{3}^{2}$ CSIRO Earth Science and Resource Engineering and Curtin University of Technology, Perth, Australia. E-mail: b.gurevich@ @urtin.edu.au.

${ }^{3}$ Curtin University of Technology, Perth,Australia.m.lebedev@ curtin.edu.au.

(C) 2010 Society of Exploration Geophysicists. All rights reserved. 
space of rocks causes attenuation and dispersion by the mechanism broadly known as wave-induced fluid flow (WIFF). WIFF occurs as a passing wave creates pressure gradients within the fluid phase and the resulting movement of the fluid relative to the solid (fluid flow) is accompanied with internal friction until the pore pressure is equilibrated. WIFF's classification depends on the length scale of the pressure gradient. If a body wave propagates through a spatially homogeneous, permeable, fluid-saturated rock, it will create pressure gradients between peaks and troughs of the wave. WIFF resulting from these wavelength-scale pressure gradients is often called global or macroscopic flow. Viscous-inertial attenuation and dispersion resulting from global flow is theoretically quantified by Biot's theory of poroelasticity (Frenkel, 1944; Biot, 1956a, 1956b, 1962; White, 1986; Bourbié et al., 1987; Pride, 2005). According to these studies, global flow attenuation in typical rocks can only be significant at frequencies above $100 \mathrm{KHz}$, well outside the seismic exploration band.

At the other end of the scale, different compliances of adjacent pores (because of the difference in their shape and/or orientation) can cause local pressure gradients between these pores. For instance, granular rocks such as sandstones are often considered to contain very compliant pores in the contact areas between adjacent grains and much larger and stiffer intergranular pores. When a seismic wave compresses a rock, the grain contact area is deformed to a much greater extent than the intergranular pores, resulting in local pressure gradients, fluid movement, and viscous dissipation. This mechanism of local or pore-scale WIFF, also known as squirt, is usually considered to be important at ultrasonic frequencies but may also play a role in seismic and logging frequencies (Johnston et al., 1979; Winkler et al., 1985; Sams et al., 1997; Pride et al., 2004).

WIFF can also occur from spatial variations in rock compliance on a scale much larger than typical pore size but much smaller than the wavelength, the so-called mesoscopic flow (Figure 1). The mechanism of WIFF at mesoscopic heterogeneities is illustrated schematically in Figure 2. In response to a passing compressional wave, the porous framework of grains is compressed and rarefied on time scales imposed by the wave speed. When heterogeneities in saturating fluids (and or rock properties) exist, the compression or rar-
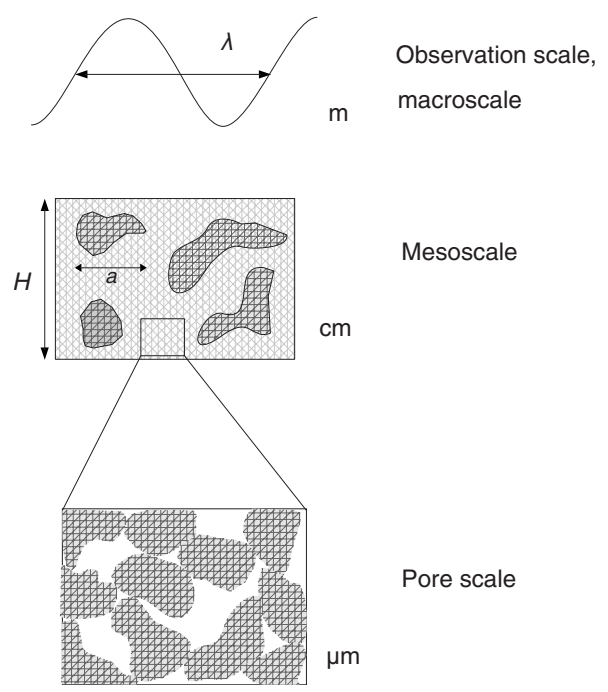

Figure 1 . The scale of heterogeneity that is typically relevant for wave-induced flow to occur at seismic frequencies is on the centimeter scale and is called mesoscopic (modified after Müller et al., 2008). efaction of the frame causes spatial gradients in fluid pressure to develop. Provided that heterogeneities exist on length scales smaller than the wavelength but greater than the pore scale, gradients in fluid pressure develop on the mesoscale. This drives the so-called mesoscopic fluid flow, which causes the attenuation of elastic energy and the dispersion of a propagating wave.

In typical sedimentary rocks, mesoscopic flow can occur within a wide range of scales, from the largest pore size to the smallest wavelength, and therefore can cause attenuation in a broad a range of frequencies. It is increasingly believed that mesoscopic flow is a significant mechanism of fluid-related attenuation in the seismic-exploration band (Pride et al., 2004). Therefore, mesocopic flow is the focus of this review. To cause significant attenuation and dispersion, the degree of heterogeneity - the contrast between elastic properties of different regions of the rock - must also be significant. There are a number of scenarios where this is manifestly the case. Two most obvious scenarios that have received substantial attention in recent years are patchy saturated rocks and fractured reservoirs.

If two immiscible pore fluids with substantially different fluid bulk moduli (such as water and gas) form patches at the mesoscopic scale, significant wave attenuation and dispersion result (Murphy, 1982). The pressure equilibration can be achieved only if the frequency is sufficiently low so that the characteristic length of fluidpressure diffusion in the pore space is large compared to the largest spatial scale of fluid mixing. If the frequency is higher, the pressure in the two fluids will not have enough time to equilibrate, resulting in a higher undrained bulk modulus and wave velocity. Hence, the presence of two fluids in the pores causes additional dispersion and attenuation of elastic waves, which is related to relaxation of porefluid pressures. The frequency dependence of wave velocity and attenuation in a partially saturated medium is controlled by the size, shape, and spatial distribution of fluid pockets; the permeability and elastic moduli of the solid matrix; and the properties of the two fluids. Following, we review related experimental works and proposed theoretical models.

A strong level of heterogeneity is also typical for fractured reservoirs such as tight sands and carbonates, where compliant mesoscopic fractures embedded in a porous rock mass play a crucial role as flow conduits (Nelson, 2001). During the compression-wave cycle, there is local flow from compliant fractures into the porous background medium and vice versa during the dilatational wave cycle. If the fractures are aligned in space, then WIFF will cause frequencydependent anisotropy. Seismic characterization of fracture sets is an important aspect of reservoir characterization; therefore, attenuation

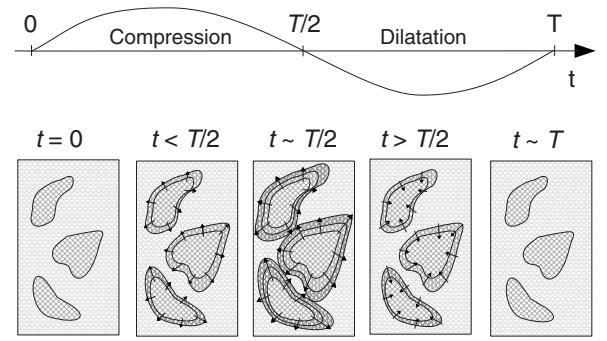

Figure 2. The mechanism of wave-induced flow. During the compression cycle of a wave with period $T$, there will be fluid flow from elastically soft inhomogeneities into the background (shown here; the flow direction is indicated by arrows) and flow from the background into elastically stiff inhomogeneities. During the extension cycle of the wave, the fluid flow reverses (modified after Müller et al., 2008). 
from WIFF and associated frequency-dependent anisotropy has attracted considerable interest (Chapman, 2003; Gurevich et al., 2009a).

Previous review papers related to fluid effects in porous rocks focus on seismic rock physics in general (Wang, 2001; King, 2009) and Gassmann fluid substitution in particular (Smith et al., 2003). Li et al. (2001) review the physics of partially saturated rocks. More recently, Toms et al. (2006a) provides a comparative review on models for wave attenuation and dispersion in partially saturated rocks. Gurevich et al. (2007, 2009a) compare different models of wave-induced flow in fractured porous media. Müller et al. (2008a) discuss the interplay between wave-induced flow attenuation and attenuation from scattering in randomly inhomogeneous porous media. In this paper, we review a number of theoretical and numerical approaches for modeling seismic attenuation and dispersion resulting from WIFF, with an emphasis on mesocopic phenomena.

The paper is organized as follows. First, we review laboratory experiments and their interpretations related to wave attenuation in porous, fluid-saturated rocks. Because the low- and high-frequency limits of WIFF can be generally described in terms of relaxed and unrelaxed elastic constants, we state the exact results for these limits for the cases of partially saturated and fractured rocks. An outline of theoretical models for attenuation and dispersion is given next. We briefly outline numerical simulation techniques related to the evaluation of frequency-dependent attenuation and dispersion in poroelastic media. A more detailed review on various numerical techniques for solving poroelasticity equations is provided by Carcione et al. (2010). We also discuss some open research problems with respect to the observability of the wave-induced flow mechanism in laboratory experiments.

\section{OBSERVATIONS AT LABORATORY AND FIELD SCALES}

A large body of experimental work addresses fluid-related wave attenuation and dispersion mechanisms in porous rock. It is often observed that intrinsic attenuation of compressional waves is frequency dependent, with a peak in the attenuation in the sonic frequency band (say, $5-30 \mathrm{kHz}$ ). This attenuation is believed to be caused by local fluid flow in pores of small aspect ratio, i.e., squirt flow (Toksöz et al., 1979; Sams et al., 1997; Adelinet et al., 2010). Local flow is influenced by several factors, such as the presence of clays (Klimentos and McCann, 1990; Best and McCann, 1995). A comprehensive review of fluid-related wave attenuation is clearly beyond the scope of this paper. Instead, we provide a brief review of papers where attenuation and dispersion are (at least in part) attributed to the presence of mesoscopic heterogeneities and where mesoscopic heterogeneities are directly observed.

In the laboratory setting, this has been done using ultrasound, resonance-bar techniques, and, more recently, dynamic stress-strain measurements. Attempts also have been made to relate field observations to WIFF. However, such interpretations of field data are ambiguous because many parameters of rocks are unknown and different dissipation mechanisms are difficult or even impossible to separate. Nevertheless, several publications consistently report indications of WIFF. Field observations, however scarce, are particularly important for mesoscopic WIFF because, in many cases, mesoscopic imhomogeneities (fractures, fluid patches) in real rocks may be larger than typical core-sample size and therefore cannot be observed in the laboratory.

\section{Laboratory experiments and interpretation}

\section{Ultrasound}

Ultrasonic velocity measurements on partially saturated rocks have been reported since the 1940s (Born, 1941; Hughes and Kelly, 1952; Wyllie et al., 1956). Gregory (1976) measures ultrasonic Pand S-wave velocities in consolidated sandstones with partial saturations of water and air and shows that the saturation dependence of the $\mathrm{P}$-wave velocity is substantially different from the effective fluid model based on Wood's (1941) mixing law, which is exact for a mixture of free fluids. Domenico (1976) observes that measured ultrasonic P-wave velocities in unconsolidated quartz sand saturated with a brine-gas mixture are higher than the values predicted by the effective fluid model and concludes that the uneven microscopic gas-brine distribution is responsible for this discrepancy.

Gist (1994) quantitatively interprets these data in terms of the gaspocket model (White model, see below), assuming the existence of mesoscopic fluid patches. Bacri and Salin (1986) report significant sound-velocity differences in an oil/brine-saturated sandstone. Depending on the experimental procedure, the saturation level changes (drainage or imbibition). Mavko and Nolen-Hoeksema (1994) and Walsh (1995) find that ultrasonic velocities are controlled by two scales of saturation heterogeneity: (1) saturation differences between thin compliant pores and larger stiffer pores and (2) differences between saturated patches and undersaturated patches at a scale much larger than any pore. These large-scale saturation patches tend to increase only the high-frequency bulk modulus by amounts roughly proportional to the saturation. Mavko and Nolen-Hoeksema (1994) conclude that patchy saturation effects can persist even at seismic field frequencies if the patch sizes are sufficiently large and the diffusivities are sufficiently low for the larger-scale pressure gradients to be unrelaxed. King et al. (2000) report patchy saturation behavior of ultrasonic velocities in sandstones.

\section{$X$-ray imaging and ultrasound combined}

Murphy et al. (1984) perform a series of experiments on sedimentary rocks using densely sampled ultrasonic phase-velocity measurements and optical, scanning-electron, and X-ray microscopy. They observe centimeter-scale heterogeneities responsible for velocity variations of up to $35 \%$. Mesoscopic fluid distributions have been directly observed in recent experiments (Cadoret et al., 1995; Cadoret et al., 1998; Monsen and Johnstad, 2005; Toms-Stewart et al., 2009). In these studies, clusters or patches of different pore fluids are distributed throughout the porous rock samples. These experiments reveal that the shape and distribution of mesoscopic fluid patches depends upon the degree of saturation and the process of fluid saturation.

X-ray microtomographic images of Cadoret et al. (1998) show that imbibition experiments, where water displaces gas, produce regular patches of fluids distributed uniformly throughout the porous rock at high water saturations. Conversely, drainage or evaporative experiments, where the reverse fluid-substitution process occurs, produce gas clusters distributed nonuniformly throughout the porous rock at high water saturations. Because drainage and imbibition produce different saturation patterns at the same level of saturation, differences in attenuation and phase-velocity measurements can be attributed to differences in fluid distribution. Moreover, phase velocities measured from drainage experiments are appreciably higher than those from imbibition experiments (Knight and Nolen- 
Hoeksema, 1990; Cadoret et al., 1995) and similarly for attenuation measurements (Cadoret et al., 1998). Thus, estimates of attenuation and phase velocity are affected by the distribution of immiscible fluids.

Recently, Lebedev et al. (2009a, 2009b) and have performed a combined fluid injection and ultrasonic experiment while monitoring fluid distribution using X-ray computer tomography. For a lowpermeability sandstone, they show that, at low saturation, the velocity-saturation dependence can be described by the Gassmann-Wood relationship (see below). At intermediate saturations, there is a transition behavior controlled by the fluid-patch arrangement and fluidpatch size that can be modeled consistently with the WIFF mechanism (Figure 3). Lei and Xue (2009) inject (gaseous and supercritical) $\mathrm{CO}_{2}$ into a water-saturated sandstone under controlled pressure and temperature conditions and monitor the saturation dependence of P-wave velocity and attenuation using a sensor array of ultrasonic transducers. They find that the velocity- and attenuation-saturation relations can be explained by WIFF, assuming patch sizes on the order of a few millimeters.

\section{Low-frequency measurements}

Although ultrasonic experiments at frequencies of $0.2-1 \mathrm{MHz}$ provide robust and accurate measurements of elastic wave attenuation, their use for calibrating field data is impeded by a large difference in frequency. To overcome this difficulty, significant efforts have been made over the years to measure acoustic properties of rocks at lower frequencies $(10 \mathrm{~Hz}-10 \mathrm{KHz})$.

Winkler and Nur (1982) perform resonance-bar experiments on sandstone samples at frequencies from 500 to $9000 \mathrm{~Hz}$ and study the effects of confining pressure, pore pressure, degree of saturation, and strain amplitude. For strain amplitudes typical of seismic wave propagation, they infer that partial saturation is a significant source of seismic P-wave attenuation in situ. They interpret attenuation in terms of wave-induced pore-fluid flow (intercrack flow; Mavko and Nur, 1979). Spencer (1981) conducts forced-deformation experi-
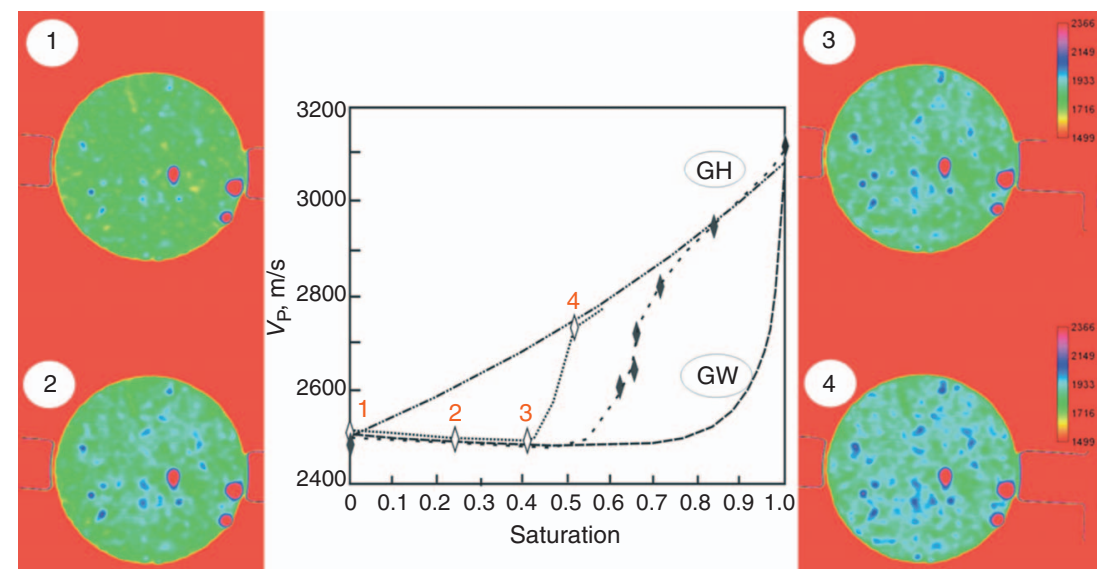

Figure 3. Ultrasonic velocity versus water saturation for the Casino Otway basin sandstone from the quasi-static saturation experiment (black diamonds) and dynamic saturation experiment (white diamonds). Numbers 1-4 indicate acoustic measurements corresponding to the computerized tomography (CT) images shown. Theoretical GassmannWood (GW) and Gassmann-Hill (GH) bounds are shown by dashed and dashed-dotted lines, respectively. CT images are of the Casino Otway basin sandstone at different times after start of injection at the same position: 1 - dried sample; $2-1$ hour; $3-24$ hours; $4-72$ hours. Contours of guide pins are visible at the right and left sides of the sample (modified after Lebedev et al., 2009b). ments at frequencies from 4 to $400 \mathrm{~Hz}$ with strain amplitudes on the order of $10^{-7}$. He finds that the frequency dependence of attenuation and dispersion in fluid-saturated sandstones and limestones can be well described by Cole-Cole distributions of relaxation times, impresence of pore fluids. (1982, 1984) uses the resonance-bar technique to infer frequency-dependent, extensional wave attenuation for various lowand high-porosity sandstones in a frequency range from $0 \mathrm{~Hz}$ to $14 \mathrm{KHz}$ with peak attenuation at approximately $85 \%$ waments on Berea sandstone during increasing (imbibition) and decreasing (drainage) brine saturation and find that extensional wave tenuation is dependent on saturation history as well as degree of variation to the size and number of mesoscale air pockets within the carbonate samples by combining forced-deformation and pulse transmission experiments. They point out that velocity dispersion and attenuation are influenced strongly by fluid mobility (the ratio of rock permeability to fluid shear viscosity). In particular, they argue that low fluid mobility may produce significant dispersion at seismic frequencies. They discuss the possibility that mesoscopic fluid mobetween heterogeneous compliant regions is the controlling at(2009) measure attenuation in find a significant increase in attenuation when brine substitutes a

\section{Field observations}

In some field-scale studies, the effect of mesoscopic heterogeneities is deduced from well-log or seismic data and where seismic attenuation or dispersion of P-waves, frequency-dependent shearwave splitting, or attenuation anisotropy are associated with the WIFF mechanism.

Sengupta et al. (2003) show that the subseismic spatial distribution of fluids not captured by traditional flow simulators can impact the seismic response. They find that downscaling smooth saturation output from a flow simulator to a more realistic patchy distribution is required to provide a good quantitative match with near- and far-offset time-lapse data, even though the fine details in the saturation distribution are below seismic resolution. Based on flow simulations of an outcrop analogue reservoir, Kirstetter et al. (2006) estimate fluid-patch sizes for different production scenarios (e.g., water displaces oil) to be on the order of $1 \mathrm{~m}$. Konishi et al. (2009) study sonic and neutron logs obtained from a $\mathrm{CO}_{2}$ sequestration test site in Japan. They find that P-wave velocity decreases gradually (almost linearly) with increasing $\mathrm{CO}_{2}$ saturation and conclude that this behavior could indicate the presence of (mesoscopic) $\mathrm{CO}_{2}$ patches. To model the velocity-saturation relation, Konishi et al. (2009) use an empirical relation suggested by Brie et al. (1995) that has also 
been used by Carcione et al. (2006) in the context of $\mathrm{CO}_{2}$ storage monitoring.

Parra (2000) reports unusually strong velocity dispersion of $\mathrm{P}$-waves (up to $30 \%$ ) and attenuation in a relatively narrow frequency range by jointly analyzing crosshole and VSP data from a limestone formation with vertical fractures embedded in a porous matrix saturated by oil and gas patches. Brajanovski et al. (2010) interprets the $\mathrm{P}$-wave dispersion as a consequence of WIFF occurring simultaneously at fractures and gas patches. Lee and Collett (2009) report Pwave velocity dispersion between surface seismic and logging frequencies on the order of $25 \%$ in gas-hydrate-bearing sediments containing free gas that is modeled using the gas-pocket model, assuming patches on the centimeter scale. Significant $\mathrm{P}$-wave attenuation resulting from WIFF between sedimentary layers with and without gas hydrates is reported by Gerner et al. (2007).

Liu et al. (2003) reveal frequency-dependent anisotropy from multicomponent VSP data in a fractured gas reservoir. They discuss two mechanisms giving rise to dispersion and frequency-dependent anisotropy: scattering of seismic waves by preferentially aligned inhomogeneneities, such as fractures or fine layers, and fluid flow in porous rocks with microcracks and macrofractures. For this data set, Maultzsch et al. (2003) analyze the frequency-dependent shearwave splitting manifested in a systematic decrease of the time delay between fast and slow shear waves with increasing frequency. They use the model of Chapman (2003) that quantifies frequency-dependent anisotropy resulting from WIFF at mesoscopic fractures to invert for fracture density and fracture radius.

By analyzing multiazimuth walkaway VSP data from a fractured hydrocarbon reservoir, Maultzsch et al. (2007) find significant azimuthal variations in P-wave attenuation at reservoir depth. They model this effect using Chapman's (2003) model for attenuation due to WIFF in the presence of aligned mesoscopic fractures. Liu et al. (2007) compare velocity and attenuation anisotropies caused by two fracture sets (one being the fluid conduit) embedded in a porous medium and find that the symmetry axes in the azimuthal variations of velocity and attenuation are significantly different. Clark et al. (2009) analyze P-wave attenuation anisotropy in the presence of a system of vertically aligned fractures revealed from multiazimuth seismic reflection data.

In conclusion, there is experimental support that the WIFF mechanism is observable in different scenarios and that its quantification has the potential to provide additional information on rock properties. To this end, theoretical models are required that accurately capture the physics of WIFF. These models are the main focus of the remainder of this review.

\section{RELAXED AND UNRELAXED LIMITS OF WAVE- INDUCED FLOW}

Because the WIFF mechanism has the characteristics of a relaxation phenomenon, it is expected that there exist effective, real-valued elastic constants (and hence velocities) representing a relaxed and unrelaxed state at very low and very high frequencies, respectively. In at least two situations, these limits can be computed exactly and therefore deserve particular attention.

\section{Patchy-saturated rocks}

In the limiting cases of very low and very high wave frequencies, theoretical values of phase velocities can be determined. For inter- mediate wave frequencies, phase velocities are frequency dependent and lie between these limiting values. Following Mavko and Mukerji (1998), Knight et al. (1998), and Johnson (2001), we present upper- and lower-frequency limits on phase velocities.

For a porous rock having only heterogeneities in saturating fluids, Norris (1993) shows that the distribution of fluid pressures is governed by a diffusion equation with diffusivity

$$
D_{p}=\frac{\kappa N}{\eta}
$$

and a diffusion length of

$$
\lambda_{p}=\sqrt{\frac{D_{p}}{\omega}},
$$

where $N$ is a poroelastic modulus defined as $N=M L / H$, with $L$ and $H$ being the P-wave moduli of the dry and fluid-saturated rock, respectively. The modulus $M$ is defined as $M=\left[\left((\alpha-\phi) / K_{g}\right)\right.$ $\left.+\left(\phi / K_{f}\right)\right]^{-1}$, with porosity $\phi$, fluid and grain bulk moduli $K_{f}$ and $K_{g}$, and the Biot-Willis coefficient $\alpha=1-K_{d} / K_{g}$, with $K_{d}$ the bulk modulus of the drained rock. In equations 1 and $2, \kappa$ denotes flow permeability, $\eta$ is the pore-fluid shear viscosity, and $\omega$ is the circular wave frequency.

When the frequency of the incident wave is sufficiently low so that the characteristic patch size of fluid heterogeneities $a$ is much smaller than the diffusion length $\lambda_{p}$,

$$
a \ll \lambda_{p}
$$

there is enough time for fluid to flow and equilibrate at a constant pressure. In this limit, Wood's (1941) law can be applied to determine an effective fluid bulk modulus given by the harmonic saturation-weighted average of the individual fluid bulk moduli $K_{f i}$ :

$$
K_{\mathrm{W}}=\left[\sum_{i=1}^{n} \frac{S_{i}}{K_{f i}}\right]^{-1},
$$

where $S_{i}$ denotes the saturation of the $i$ th fluid and the subscript W stands for Wood. Once the effective bulk modulus of the pore fluid is defined, Gassmann relations can be applied to estimate the low-frequency phase velocity for a partially fluid-saturated rock. The resulting P-wave modulus is

$$
H_{\mathrm{GW}}=L+\alpha^{2} M\left(K_{\mathrm{W}}\right),
$$

where $M\left(K_{\mathrm{W}}\right)$ means that equation 4 is used to compute the modulus $M$ and where the subscript GW refers to Gassmann-Wood. This quasi-static limit is known as uniform saturation or the Gassmann-Wood limit.

Conversely, when the wave frequency is sufficiently high and the characteristic patch size is larger than the diffusion length,

$$
a \gg \lambda_{p}
$$

there is not enough time for pressure equilibration, and fluid-flow effects can be ignored. In this circumstance, patches of rock will remain at different pressures. Applying Gassmann's theory to individual patches allows the saturated bulk modulus of each patch to be determined. According to Gassmann's equations, the saturated shear modulus of each patch is independent of fluid bulk modulus. Thus, Hill's (1963) theorem can be applied to determine the overall saturated bulk modulus via harmonic averaging of the $\mathrm{P}$-wave moduli: 


$$
H_{\mathrm{GH}}=\left[\sum_{i=1}^{n} \frac{S_{i}}{H\left(K_{f i}\right)}\right]^{-1},
$$

where $H\left(K_{f i}\right)$ denotes the P-wave modulus computed for the $i$ th fluid bulk modulus and where the subscript GH stands for GassmannHill.

This high-frequency or no-flow limit is known as the patchy or Gassmann-Hill limit. The elastic moduli in the low- and high-frequency limits are given by real numbers and are frequency independent. Johnson (2001) shows that for any nonzero saturation, the Gassmann-Wood moduli (corresponding to homogeneous saturation) are always smaller than those for Gassmann-Hill moduli (corresponding to patchy saturation). He also shows that at intermediate frequencies, the bulk modulus lies between these limits. Thus, for any intermediate saturation level, the phase velocity is a monotonically increasing function of frequency.

\section{Mesoscopic fractures}

Many reservoir rocks such as carbonates or tight sands contain voids of two types: stiff voids such as pores or vugs (so-called equant porosity; Thomsen, 1995), which contain most of the fluids, and fractures whose overall volume (fracture porosity) is small (often less than $0.1 \%$ ). In the simplest case of fully aligned, hydraulically isolated and rotationally symmetric fractures, the effect of fractures on the overall elastic behavior of the reservoir can be quantified using two parameters: normal and tangential excess fracture compliances $Z_{N}$ and $Z_{T}$ (Schoenberg, 1980). When fractures are dry or filled with gas, $Z_{N}$ and $Z_{T}$ are the same order of magnitude (Schoenberg and Sayers, 1995). When the fractures are filled with a liquid (say, brine or oil), normal fracture compliance $Z_{N}$ vanishes and P-wave velocities parallel and perpendicular to fractures become almost equal. Such behavior is caused because the liquid drastically stiffens the otherwise very compliant fractures. They are hydraulically isolated, and the liquid has nowhere to go.

Fluid-saturated fractured rocks with equant porosity represent an intermediate case between dry and liquid-filled rocks with isolated fractures. When such a rock is compressed perpendicularly to the fracture plane, the fluid can escape into the equant pores, thus increasing the normal fracture compliance. This is a first-order effect; hence, any model of the fractured reservoir with equant porosity must take this fluid flow into account. The effect was first quantified by Thomsen (1995) for a specific case of sparse penny-shaped cracks and spherical equant pores. More recently, Gurevich (2003) shows that exact static elastic moduli of a fluid-saturated fractured rock with equant porosity can be obtained directly from the properties of unfractured porous rock and fracture compliances of the dry fractured rock using anisotropic Gassmann (1951) or Brown-Korringa (1975) equations (see also Berryman, 2007). This approach is completely general and does not require one to specify the shape of pores or fractures as long as their cumulative effect on the properties of the dry rock is known.

Thomsen's equant porosity theory and the anisotropic Gassmann approach assume that the frequency of the elastic wave is sufficiently low to attain so-called relaxed conditions, where fluid pressure is equal in pores and fractures. This occurs when the fluid diffusion length is large compared to the characteristic dimensions of the fracture system. At the other end of the frequency spectrum, the fluid diffusion length is small compared to characteristic fracture size, and the fluid has no time to move between pores and fractures during a wave cycle. Thus, the fractures behave as if they were isolated (unrelaxed regime; see Mavko and Jizba, 1991; Endres and Knight, 1997; Berryman, 2007; Gurevich et al., 2009b). The predictions in the unrelaxed limit have been observed to be consistent with laboratory measurements at ultrasonic frequencies (Mavko and Jizba, 1994; Wulff and Burkhardt, 1997).

In principle, a general treatment of the relaxed and unrelaxed limits for a large class of heterogeneity shapes and distributions can be based on effective medium theory (O'Connell and Budiansky, 1974; Berryman, 1980a, 1980b; Guéguen and Palciauskas, 1994; Milton, 2002). For example, O'Connell and Budiansky (1974) calculate the variability of the effective elastic moduli for fluid-filled cracked solids as a function of the fluid bulk modulus, thus identifying a way to model relaxed and unrelaxed limits. The difference between the relaxed (low-frequency) and unrelaxed (high-frequency) moduli of rock volumes with different compliances and where fluid communication is generally possible implies that WIFF will occur at intermediate frequencies. At these frequencies, WIFF causes attenuation, dispersion, and frequency-dependent anisotropy.

\section{FREQUENCY-DEPENDENT ATTENUATION AND DISPERSION}

The relaxed and unrelaxed elastic moduli are exactly known for the scenarios discussed above and result in simple expressions for the $\mathrm{P}$ - and $\mathrm{S}$-wave velocities. For finite frequencies, however, the description of attenuation and velocity dispersion attributable to WIFF requires solving a dynamic problem. More precisely, a coupled elastic (to quantify the wave-induced fluid pressure) and hydrodynamic (to model fluid-pressure equilibration) problem must be solved for an inhomogeneous solid. For several particular inhomogeneity geometries (and arrangements in space), analytical solutions for attenuation and phase-velocity dispersion are feasible. We review some of these approaches, focusing on the theoretical approach taken in different space dimensions. Although many models have been developed using poroelasticity theory, other models are based on elastodynamic theory incorporating fluid flow through communicating cavities.

\section{Models based on Biot's theory of poroelasticity}

Gassmann equations define elastic wave velocities in fluid-saturated porous media in the low-frequency limit. These equations are widely used in the petroleum industry for estimating seismic wave velocities in hydrocarbon reservoirs (Wang, 2001; Smith et al., 2003; Mavko et al., 2009). However, at finite frequencies, seismic wave propagation may violate the quasi-static assumption, causing deviations from Gassmann results. In particular, wave attenuation and phase-velocity dispersion cannot be modeled with Gassmann equation.

To account for these effects, Biot's theory is often utilized. Biot's equations (Biot, 1956a, 1956b, 1962; see also Bourbié et al., 1987; Allard, 1993; and Carcione, 2007) of dynamic poroelasticity provide a general framework for modeling elastic wave propagation through porous, fluid-saturated media. The equations were derived using a Lagrangian viewpoint with generalized coordinates given by the solid and fluid displacements averaged over a certain representative volume element (RVE) of a porous medium. A dissipation function is introduced, which depends only upon relative solid and fluid motion. There are four basic assumptions of Biot's equations of po- 
roelasticity. First, the porous rock frame is homogeneous and isotropic. It has uniform porosity, frame bulk modulus, shear modulus, density, and permeability, and it consists of only one grain type, characterized by bulk modulus, shear modulus, and density. Second, the porous rock is fully saturated by only one fluid having shear viscosity, fluid bulk modulus, and fluid density. Third, relative motion between solid and fluid is governed by Darcy's law. And last, the wavelength of the passing wave is substantially larger than the size of the largest grains or pores.

Biot's theory predicts the existence of a second, slow P-wave that is highly attenuative at low frequencies and propagating without loss at high frequencies. The transition from the viscosity-dominated regime to the inertial regime is often modeled using the dynamic permeability model of Johnson et al. (1987). For sufficiently low frequencies, i.e., lower than Biot's characteristic frequency,

$$
\omega_{\mathrm{Biot}}=\frac{\eta \phi}{\rho_{f} \kappa \alpha_{\infty}},
$$

with fluid density $\rho_{f}$ and tortuosity $\alpha_{\infty}$, fluid flow within the pore channels is approximated as Poiseuille flow. This means the flow is laminar (i.e., the Reynolds number of the flow that expresses the ratio of inertial forces to viscous forces is less than a critical Reynolds number) and the dynamic permeability is a real-valued constant. For most rocks, Biot's characteristic frequency $f_{c}=\omega_{\text {Biot }} / 2 \pi$ turns out to be about $100 \mathrm{KHz}$ or higher. Therefore, for most seismic applications, the low-frequency version of Biot's theory is adequate.

In the low-frequency regime, the Biot slow wave degenerates to a diffusion wave with the wavenumber (Norris, 1993):

$$
k_{P 2}=\sqrt{\frac{i \omega}{D_{p}}} .
$$

A diffusion equation for the fluid mass derived from the dynamic equations of poroelasticity (Biot, 1962) has been obtained by Dutta and Odé (1979a) and Chandler and Johnson (1981), confirming the equivalence between quasi-static flow and Biot's slow wave. In these works, the limit of zero frequency is constructed by strictly neglecting all inertial terms such that no fast-wave modes exist. This approach yields the same diffusion equation as previously derived from the theory of static poroelasticity (Rice and Cleary, 1976; Rudnicki, 1986). The general properties of diffusion waves are discussed by Mandelis (2000).

The importance of Biot's slow wave for permeability estimation in reservoir geophysics is investigated by Shapiro et al. (2002). In their approach, a fluid injection induces pressure perturbations (slow waves) that diffuse through the rock mass and trigger microearthquakes, provided the pressure-perturbation magnitude exceeds the rock (failure) criticality. The analysis of attenuation resulting from WIFF in the framework of Biot's theory can be thought of as the conversion (at interfaces or on spatial heterogeneities of the porous medium) between the propagating (fast) wave modes and the diffusive slow-wave mode.

\section{Interlayer flow - 1D dynamic-equivalent-medium models}

Interlayer flow, i.e., WIFF in a sequence of poroelastic layers, occurs when the fluid is squeezed from more compliant layers into stiffer layers during the compressional cycle of a wave, and vice versa. This $1 \mathrm{D}$ problem has attracted considerable attention because it is the simplest problem and is readily amenable to theoretical analysis.
Also, thin layering is the most common and well-understood type of heterogeneity in the sedimentary crust.

White et al. (1976) compute a frequency-dependent complex $\mathrm{P}$-wave modulus for a periodically stratified medium by considering the ratio of the imposed pressure amplitude to the corresponding fractional change in volume (including effects of fluid flow). Results identical to those of White et al. (1976) are reproduced using Biot's equations of dynamic poroelasticity with periodic coefficients (Norris, 1993; Carcione and Picotti, 2006). The complex P-wave modulus for a periodic system of two layers with thicknesses $L_{1}, L_{2}$, and spatial period $\Gamma=L_{1}+L_{2}$ is obtained as

$$
\tilde{H}(\omega)=\left[\bar{H}^{-1}+\frac{2\left(\frac{\alpha_{2} M_{2}}{H_{2}}-\frac{\alpha_{1} M_{1}}{H_{1}}\right)^{2}}{i \omega \Gamma \sum_{i=1}^{2} \eta_{i} /\left(\kappa_{i} k_{P 2 i}\right) * \operatorname{coth}\left(\frac{k_{P 2 i} L_{i}}{2}\right)}\right],
$$

where the subscripts refer to the properties of phase 1 and phase 2 and where $\bar{H}=\left(L_{1} /\left(\Gamma H_{1}\right)-L_{2} /\left(\Gamma H_{2}\right)\right)^{-1}$. If only the fluid bulk modulus of phase 1 differs from that of phase 2, we have $\widetilde{H}(\omega=0)$ $=H_{\mathrm{GW}}$ and $\widetilde{H}(\omega=\infty)=H_{\mathrm{GH}}$. The asymptotic scaling of attenuation for this model yields expected, physically consistent results. At low frequencies, P-wave attenuation (in terms of quality factor $Q^{-1}$ ) scales linearly with wave frequency:

$$
Q_{\text {periodic }}^{-1} \propto \omega \text {. }
$$

At high frequencies, it scales as

$$
Q^{-1} \propto \omega^{-1 / 2} .
$$

Brajanovski et al. (2005) consider a periodic medium with very thin and compliant layers embedded in a poroelastic background in order to model the effect of fractures parameterized with the linear slip theory. Brajanovski et al. (2006) show that these fractures give rise to an intermediate low-frequency regime where the quality factor scales as

$$
Q_{\text {inter }}^{-1} \propto \omega^{1 / 2} .
$$

This means that in periodically stratified media, three attenuation scaling laws are possible (equations 11-13). Indeed, for a model of periodically alternating elastically stiff, thick layers (background rock) and compliant, thin layers (fracture), Müller and Rothert (2006) compute P-wave attenuation by determining the amount of dissipated energy during a wave cycle, assuming the compression cycle of the wave instantaneously induces a constant, elevated fluid pressure in the more compliant layer that is then equilibrated through pressure diffusion.

The resulting frequency dependence of wave attenuation is identical to that obtained for the periodic fracture model of Brajanovski et al. (2006) and is displayed in Figure 4. The transition frequency $\omega_{c}$ separating the $\omega$ and $\omega^{1 / 2}$ regimes is proportional to the ratio of pressure diffusivity and square of the thickness of the stiff layer (denoted by subscript 2 ):

$$
\omega_{c} \propto \frac{D_{2}}{L_{2}^{2}} .
$$

Maximum attenuation is attained at 


$$
\omega_{\max } \propto \frac{e_{2}^{2}}{e_{1}^{2}+e_{1} e_{2}} \frac{D_{1}}{L_{1}^{2}},
$$

where subscript 1 denotes the properties of the soft layer and $e_{i}$ $=\kappa_{i} /\left(\eta_{i} \sqrt{D_{i}}\right)$ is the effusivity (a measure of the ability to interact with other phases via pressure diffusion). The separation of these crossover frequencies depends on the ratios of spatial scales and poroelastic moduli:

$$
\frac{\omega_{\max }}{\omega_{c}} \approx\left(\frac{N_{1}}{N_{2}}\right)^{2}\left(\frac{L_{2}}{L_{1}}\right)^{2} .
$$

If the assumption of periodic stratification is relaxed but instead the layer properties are assumed to fluctuate randomly with depth (or the layer thickness itself becomes a random variable), one can still use Biot's equation of poroelasticity with the coefficients of the system of partial-differential equations as random variables. Using statistical smoothing, Lopatnikov and Gurevich (1986) and Gurevich and Lopatnikov (1995) derive a complex P-wavenumber of a dynamic-equivalent medium. Interestingly, for random layering, the low-frequency asymptotic attenuation scaling is proportional to $\sqrt{\omega}$ (equation 10). This result implies that the WIFF mechanism in random media (1) plays a role in a broader frequency range compared to periodic media and (2) does not produce the attenuation proportional to $\omega$ at any frequencies. Maximum attenuation (and thus strongest change in phase velocity) is attained at

$$
\omega_{\max } \propto \frac{D_{p}}{a^{2}},
$$

where $a$ is the correlation length of the random fluctuations. For a typical water-saturated reservoir sandstone ( $\kappa=150 \mathrm{md}, \phi=0.15)$ and a correlation length of $10 \mathrm{~cm}$, maximum attenuation is predicted at $f_{\max }=\omega_{\max } / 2 \pi=25 \mathrm{~Hz}$.

Gelinsky and Shapiro (1997) and Gelinsky et al. (1998) generalize these results in the framework of the extended O'Doherty-Anstey formalism, treating WIFF and elastic scattering on an equal footing. Müller and Gurevich (2004) adapt the 1D random-medium

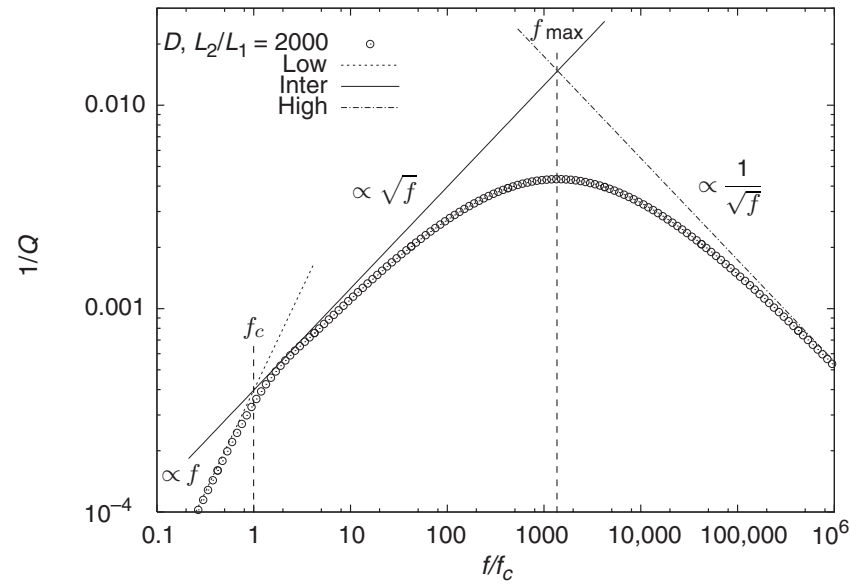

Figure 4. Frequency-dependent attenuation from interlayer flow. Three asymptotic regimes can be clearly identified; at low frequencies, $1 / Q$ scales with $f=\omega / 2 \pi$, whereas at high frequencies it scales with $1 / \sqrt{\omega}$. There is an intermediate regime where $1 / Q$ scales with $\sqrt{\omega}$. The normalization frequency is defined through equation 14 (after Müller and Rothert, 2006). model for the particular case of patchy saturation and interpret the laboratory data of Murphy (1984) and Cadoret et al. (1998). Alternative formulations of attenuation from interlayer flow are developed by Markov and Yumatov (1988) and Auriault and Boutin (1994).

\section{Discrete inclusion-based models in 3D space}

White (1975) analyzes attenuation and dispersion for a periodic arrangement of concentric spheres in a fashion similar to the periodically layered model. This model has been revised and reformulated in the context of Biot's theory of poroelasticity by Dutta and Odé (1979a, 1979b) and Dutta and Seriff (1979). It is perhaps the most widely used model for interpreting measurements in partially or patchy saturated rocks and for verifying other models or numerical simulations. The model shows the same asymptotic scaling as the analogous interlayer flow model (White et al., 1976). The characteristic frequency separating relaxed and unrelaxed regimes is

$$
\omega_{\max } \propto \frac{D_{2}}{b^{2}}
$$

where subscript 2 refers to properties of the outer shell and $b$ is the radius of the outer shell.

Walsh (1995) analyzes seismic attenuation in partially saturated rocks and argues that at higher (water) saturations - say, $>50 \%$ attenuation is caused by local flow from completely saturated regions, composed of many pores, to dry regions. He derives a complex bulk modulus, consistent with the Gassmann-Wood and Gassman-Hill moduli at low and high frequencies, respectively, where the frequency dependence is evaluated by solving the fluid-pressure-diffusion equation for the spherical shell geometry in analogy to White (1975). Attempts to overcome the assumptions of the White model, i.e., the spatial periodicity and single-sized spheres, have been developed using effective-medium theory (le Ravalec et al., 1996; Taylor and Knight, 2003).

Ciz et al. (2006) derive explicit expressions for attenuation and phase-velocity dispersion resulting from a random distribution of spherical heterogeneities within porous rock. First, the problem of scattering by a single inclusion is analyzed. Under the assumption of mesoscopic inclusion, this analysis yields a closed-form solution for the scattering amplitude (Ciz and Gurevich, 2005). The second stage utilizes Waterman and Truell's (1961) theorem of multiple scattering to approximate the scattered wavefield of a system of randomly distributed poroelastic inclusions. The discrete random model discussed here is limited to spherical patches and in this respect is similar to the regular patch model of White (1975). Unlike perodic models, however, the discrete random model is based on scattering theory and thus implies spatially random distribution of the fluid patches. On the other hand, the phase-velocity and attenuation estimates are based on the single scattering approximation of Waterman and Truell (1961), so they are limited to small concentrations of inclusions, especially when the contrast between the host and the inclusion is high. This limitation is particularly severe when describing the problem of gas patches in a liquid saturated host. Thus, the model of random spherical patches provides a good test case for other theoretical and numerical models. This is useful for small concentrations of inclusions because it gives closed-form frequency dependence of attenuation and dispersion for arbitrary contrast between inclusions and host (Toms et al., 2006a, 2007).

A natural way to generalize the discrete random model is to extend it to ellipsoidal patches. Potentially, this can yield a very versatile 
model because oblate spheroids can be used to model compliant secondary porosity, including fractures. However, to date the extension to ellipsoids has only been successful in the Born approximation (Gurevich et al., 1998), which is limited to small contrasts between host and inclusions and therefore is not applicable to fractures or, indeed, to gas patches.

Although extension of the discrete random model with spherical inclusions to arbitrary ellipsoids proves difficult, it has been more successful at the other end of the spectrum - to strongly oblate penny-shaped mesoscopic fractures whose thickness is much smaller than the fluid-diffusion length. The overall approach is broadly similar to the one for spheres. First, the problem of the interaction of a plane elastic wave with a single crack is analyzed (Galvin and Gurevich, 2007). For a fracture of mesoscopic radius and infinitesimal thickness, the analysis gives a linear integral equation for the scattering amplitude. Then the attenuation and dispersion are obtained by applying the Waterman-Truell (1961) scattering approximation (Galvin and Gurevich, 2009). This theory gives simple expressions for the low- and high-frequency asymptotic behavior of velocity and attenuation; however, at intermediate frequencies, the integral equation must be solved numerically.

\section{$3 D$ random-continuum models}

Discrete inclusion models have been obtained for regular shapes of inclusions, such as spheres, ellipsoids, or penny-shaped fractures. An alternative class of models can be developed by assuming that (poro-)elastic-medium parameters fluctuate randomly in space. These random media are parameterized using statistical moments. Consequently, instead of solving a boundary-value problem, one must solve the governing partial differential equations (PDEs) in which the coefficients become random fields. A number of techniques exist to solve such stochastic PDEs (Rytov et al., 1989).

Müller and Gurevich (2005a) model wave propagation in a 3D inhomogeneous porous medium using the low-frequency version of Biot's equations of poroelasticity with randomly varying coefficients. By using a Green's function approach, these partial-differential equations transform into a system of integral equations. The latter system is solved by means of the method of statistical smoothing, which is widely used in problems of electromagnetic, acoustic, and elastic wave propagation (Karal and Keller, 1964). More precisely, a first-order statistical smoothing approximation is used, sometimes referred to as a Bourret approximation. With this strategy, Müller and Gurevich (2005a) analyze the conversion scattering from fast $\mathrm{P}$ waves into Biot's slow wave in $3 \mathrm{D}$ randomly inhomogeneous porous media. Biot's slow wave is a highly dissipative wave mode. Therefore, the use of the first-order statistical smoothing approximation to the conversion scattering problem in Biot's equations of poroelasticity quantifies the dissipation of wavefield energy attributable to energy transfer from the coherent component of the fast $\mathrm{P}$-wave into the dissipative slow $\mathrm{P}$-wave mode.

This method differs from the usual application of smoothing to energy-conserving wave systems, where an apparent dissipation scattering attenuation - results from the energy transfer from the coherent component of the wavefield into the incoherent (scattered) component. The result of this theory is an expression for an effective, dynamic-equivalent $\mathrm{P}$-wavenumber:

$$
\tilde{k}_{\mathrm{P}}(\omega)=k_{\mathrm{P}}\left(1+\Delta_{2}+\Delta_{1} k_{\mathrm{P} 2}^{2} \int_{0}^{\infty} r B(r) \exp \left[i k_{\mathrm{P} 2} r\right] d r\right),
$$

where $\Delta_{1}$ and $\Delta_{2}$ are linear combinations of the variances of the fluctuating medium parameter and $B(r)$ is the corresponding variancenormalized correlation function. In the absence of medium-parameter fluctuations $\left(\Delta_{1}=\Delta_{2}=0\right)$, an undisturbed P-wave with wavenumber $k_{\mathrm{P}}$ propagates through the poroelastic solid. The results are based on perturbation theory, so their validity is restricted to weakcontrast media.

In Figure 5, attenuation and dispersion are shown for different correlation functions. The low-frequency behavior of attenuation is

$$
Q_{\text {random }}^{-1} \propto \omega .
$$

These asymptotes coincide with those predicted by the periodicitybased approaches. Consequently, in 3D space, the observed frequency dependence of attenuation from fluid flow has universal character
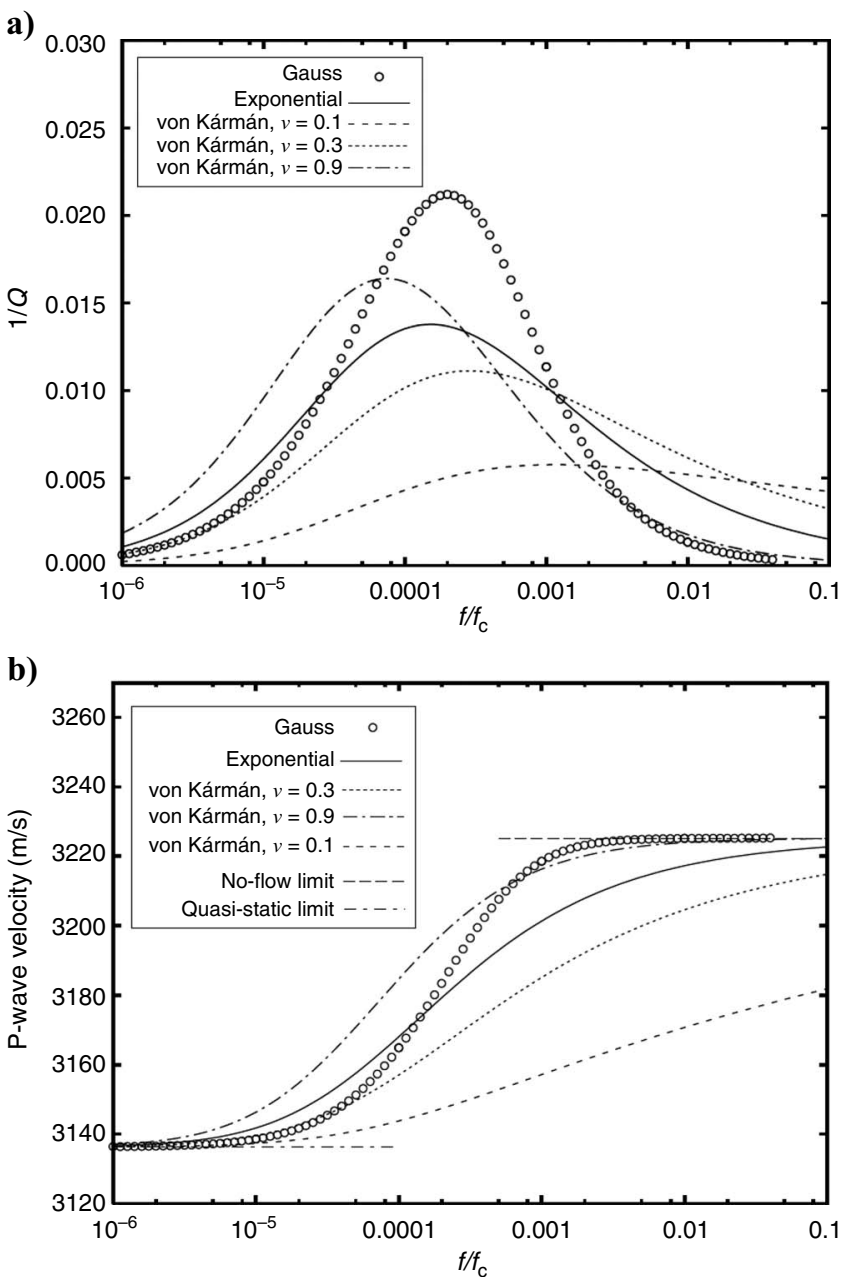

Figure 5. (a) Attenuation and (b) P-wave velocity predictions of the $3 \mathrm{D}$ continuum random medium model as a function of normalized frequency for Gaussian, exponential, and von Kármán (the Hurst coefficient $\nu$ is denoted in the legend) correlation functions (modified after Müller and Gurevich, 2005b). 
independent of the type of disorder — periodic or random. This result is somewhat unexpected if we remember that in 1D space, the attenuation asymptotes are different for periodic and random structures (see also Müller and Rothert, 2006).

The high-frequency asymptote has no universal scaling but depends on the type of correlation function and/or the fractal dimension of the heterogeneities (Shapiro and Müller, 1999; Masson and Pride, 2006; Müller et al., 2008b). For example, assuming a correlation function of the form $B(r)=\exp (-|r / a|)$ results in an asymptotic high-frequency scaling $Q^{-1} \propto \omega^{-1 / 2}$ (identical to the result for the periodicity-based models; equation 12), whereas the Gaussian correlation function $B(r)=\exp \left(-r^{2} / a^{2}\right)$ gives $Q^{-1}$ $\propto \omega^{-1}$. That is to say, the high-frequency asymptotic attenuation scaling is controlled by the smoothness of the transition region between neighboring mesoscopic heterogeneities over which the wave-induced fluid pressure is equilibrated.

This behavior is demonstrated numerically by Masson and Pride (2007), who model the effect of a transition layer of thickness $d$ embedded between two mesoscopic patches with different poroelastic moduli. For large $\lambda_{p} / d$, the fluid-pressure equilibration is sensitive to the abrupt change of patch properties. Thus, pressure diffusion occurs in two homogeneous patches that are in hydraulic connection, resulting in $Q^{-1} \propto \omega^{-1 / 2}$ scaling. For small $\lambda_{p} / d$, the fluid-pressure equilibration is confined to the transition layer with gradually changing properties, and thus $Q^{-1} \propto \omega^{-1}$.

The model of Müller and Gurevich (2005b) is restricted to mesoscopic inhomogeneities $a \geq a_{\text {pore }}$, where $a_{\text {pore }}$ denotes any characteristic pore-scale length. If the seismic wavelength is much larger than $a$, attenuation from scattering on weak inhomogeneities is negligible. However, if $\lambda \approx a$, scattering attenuation becomes noticeable. Such a case may occur at sonic or ultrasonic frequencies. Scattering attenuation can also be modeled in the framework of the statistical smoothing method. However, because the latter approximation describes signatures of the ensemble-averaged field, its application to the evaluation of scattering attenuation in heterogeneous rocks is limited. Instead, a wavefield approximation valid in single realizations of the random medium should be used. The analysis of elastic scattering is beyond the scope of this paper; for an in-depth discussion, see Müller et al. (2008a).

The continuum random media approach has been adapted to the case of patchy saturation, i.e., if only contrasts in the pore-fluid properties exist (Toms et al., 2007) and if the patchy pore-fluid distribution forms a band-limited fractal (Müller et al., 2008b). We believe the random-continuum models are particularly useful for quantifying the saturation dependence of attenuation and velocity in patchy saturated porous media.

\section{Branching-function approaches}

Analysis of the frequency dependence of attenuation and dispersion for arbitrary inclusion shapes or distributions is challenging because the corresponding boundary value problems become cumbersome. It is therefore expedient to develop approaches that make explicit use of the exact low- and high-frequency limits (see previous section) or, even more advanced, to use the known asymptotic scaling at low and high frequencies and then connect these asymptotes with a simple branching function. The choice of branching function should be based on the general causality principle, imposing a particular relationship between attenuation and dispersion (Mavko et al., 2009).
Johnson (2001) develops a theory for the acoustics of patchy saturation within the context of the low-frequency Biot theory. The dynamic bulk modulus $\tilde{K}(\omega)$ of a partially fluid-saturated porous rock is developed by first considering its response to low- and high-wave frequencies. When wave frequencies are sufficiently low, the rock is relaxed because fluid pressure is equilibrated. In this limit, the lowfrequency asymptote of the dynamic bulk modulus converges to the Gassmann-Wood limit $K_{\mathrm{GW}}$. Conversely, when wave frequencies are sufficiently high, the rock is unrelaxed because fluid pressures are unequilibrated. In this limit, the high-frequency asymptote of the dynamic bulk modulus converges to the Gassmann-Hill limit $K_{\mathrm{GH}}$. For intermediate frequencies, the dynamic response of the porous rock is constructed using a branching function, which ensures causality of the solution and convergence to higher and lower limits, similar to the approach used by Johnson et al. (1987):

$$
\tilde{K}(\omega)=K_{\mathrm{GH}}-\frac{K_{\mathrm{GH}}-K_{\mathrm{GW}}}{1-\zeta+\zeta \sqrt{1-\frac{i \omega \tau}{\zeta^{2}}}},
$$

where $\zeta$ and $\tau$ are functions of the poroelastic parameters and the geometry of the fluid patches. In this formulation, two new geometrically significant parameters are introduced: the specific surface area of the inclusion/composite volume and an effective patch-size parameter.

Using this theory, Tserkovnyak and Johnson (2001) deduce values for the specific surface area and effective patch size from experimental data. They find that their theory can be used to interpret geometric measures of partial fluid saturation from attenuation and phase-velocity measurements. Tserkovnyak and Johnson (2003) extend Johnson's (2001) patchy saturation theory by including capillary forces in the analysis, resulting in a change of the GassmannWood modulus. Toms (2008) demonstrates how Johnson's (2001) patchy saturation theory can be applied to arbitrary complex patch shapes. To this end, the determination of the shape parameter $\zeta$ requires the numerical evaluation of (1) a potential equation via the finite-difference technique and (2) the specific surface area using Monte Carlo simulations.

A more general approach based broadly on similar principles has been developed by Pride and Berryman (2003a, 2003b). Their approach yields estimates of attenuation and phase velocity in a general double-porosity, dual-permeability medium. The theory utilizes Biot's equations of poroelasticity to determine the poroelastic response of a composite body comprising two distinct poroelastic phases. Pride et al. (2004) customize the general results of Pride and Berryman (2003a, 2003b) to the specific cases of patchy saturation, where only heterogeneities in saturating fluids exist, and squirt-flow attenuation. The results for the patchy saturation case are very similar to the results of Johnson (2001).

Central to the double-porosity, dual-permeability theory is a model for fluid transport between two poroelastic phases in which the induced fluid pressures are different. Fluid flow between phases is assumed to be proportional to the induced pressure difference with a frequency-dependent proportionality coefficient:

$$
-i \omega \xi=\tilde{\gamma}(\omega)\left(p_{1}-p_{2}\right),
$$

where $\zeta$ is the fluid increment $(-i \omega \xi$ denotes the rate of transferred fluid volume) and $p_{i}$ refers to the average fluid pressure in the $i$ th phase. First, this fluid transport coefficient $\tilde{\gamma}(\omega)$ is determined for low and high frequencies. Then, following an approach of Pride et al. 
(1993), a branching function is introduced that links low- and highfrequency limits to determine the overall frequency dependence of the fluid-transport coefficient:

$$
\tilde{\gamma}(\omega)=\gamma_{m} \sqrt{1-\frac{i \omega}{\omega_{m}}},
$$

with the parameters $\gamma_{m}$ and $\omega_{m}$ depending on the particular mesoscopic geometry (and elastic properties).

For the case of patchy saturated rocks modeled as periodically spaced concentric spheres, the Pride-Berryman, Johnson, and White models are compared in Figure 6. The characteristic frequency (for maximum attenuation) is given by

$$
\omega_{\max }=\frac{K_{d} B_{1}}{\eta_{1} \alpha} \frac{\kappa\left(\frac{v_{1} V}{S}\right)^{2}}{L_{1}^{4}}\left(1+\sqrt{\frac{\eta_{2} B_{2}}{\eta_{1} B_{1}}}\right)^{2},
$$

where $S / V$ denotes the specific surface of the fluid patches and subscripts 1 and 2 refer to the two different phases, with volume fraction of phase 1 , denoted as $v_{1}$. The value $B_{i}$ is Skempton's coefficient, and $L_{1}$ is a characteristic length scale over which the fluid-pressure gradient still exists (in phase 1). It is important to note that using equations 21 or 23 always results in the asymptotic attenuation scaling laws 11 and 12 at low and high frequencies, respectively.

All of the above approaches are limited to modeling attenuation and phase-velocity dispersion from WIFF arising between fluid heterogeneities that are identical and distributed regularly throughout the porous medium. This consequence is the result of decomposing the porous medium into identical cells (or composite volumes) containing fluid heterogeneities of regular shape. Nevertheless, we believe that the branching function provides a versatile concept to quantify attenuation and dispersion. Interestingly, the causality constraint for the branching function captures the physics of WIFF very well. In fact, some of the inclusion-based and random-continuum models can be represented accurately in terms of a branching function, facilitating the comparison between effects from different types of heterogeneities and creating simple approximations.

The branching function approximation has been derived for cases

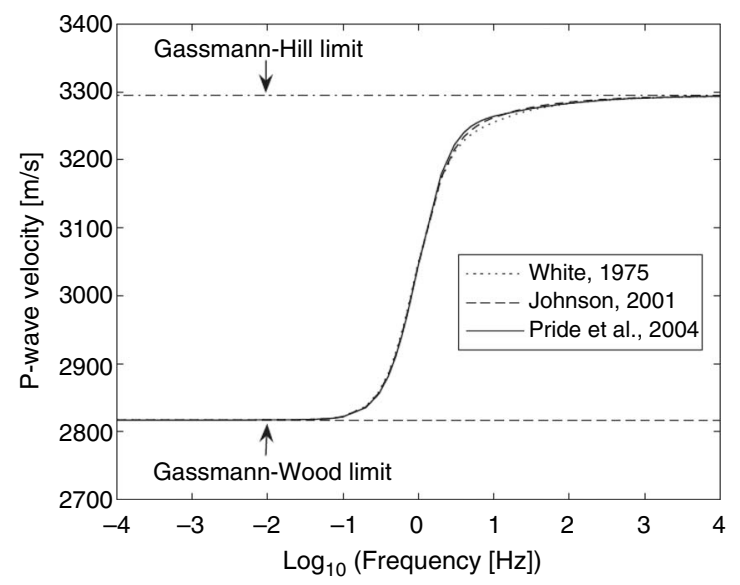

Figure 6. Phase-velocity dispersion predicted by the periodic models of White (1975), Johnson (2001), and Pride et al. (2004). There is very good agreement between all approaches for the case of 5\% air inclusions in an otherwise water-saturated host rock of $15 \%$ porosity. The inclusion radius is $25 \mathrm{~cm}$ (modified after Toms et al., 2006a). of a concentric-spheres model of White by Johnson (2001) for a periodic distribution of concentric circles by Krzikalla et al. (2006), for the 3D random patchy saturation model of Toms et al. (2007) by Toms et al. (2006b), and for periodically spaced planar and aligned fractures by Gurevich et al. (2009a). The behavior of the attenuation and dispersion in equation 21 is controlled by two parameters: $\zeta \geq 0$ and $\tau>0$. Parameter $\zeta$ controls the shape of the attenuation and dispersion curves, and $\tau$ defines the time scaling. Similarly to equation 10, equation 21 (and also equation 23) allows us to define, in general, up to three different attenuation/dispersion regimes characterized by $\omega \tau \leq \zeta^{2}, \zeta^{2} \leq \omega \tau \leq 1$, or $\omega \tau \geq 1$. These three regimes are separated by two characteristic frequencies. For example, for periodically spaced planar fractures, Gurevich et al. (2009a) deduce

$$
\omega_{c}=\frac{2 \zeta^{2}}{\tau}, \quad \omega_{\max }=\frac{1}{\tau},
$$

which are analogous to frequencies 14 and 15 derived above. The existence of the crossover frequency $\omega_{c}$ has not been noticed in earlier works using the branching-function ansatz. However, we believe this is an important feature of wave-induced diffusion processes.

\section{Models using hydraulically communicating cavities}

In the equations of poroelasticity used in the models described in the preceding section, the shape of various pores is not defined explicitly but rather by integral parameters of the RVE, such as porosity, permeability, and bulk and shear moduli of the dry matrix. An alternative approach is to consider pressure relaxation between pores or cavities of different shapes. This latter approach is particularly useful for modeling pore-scale phenomena (where Biot's RVE concept is not applicable) or in problems where pore shapes can or need to be defined explicitly (such as in man-made materials). It builds upon a substantial body of work on effective elastic properties of elastic media with a distribution of cavities (isolated or connected) of different shapes and orientations.

\section{Squirt flow}

Local or squirt flow refers to the mechanism of viscous dissipation resulting from flow within one pore of nonspherical shape or between adjacent pores of different shapes and/or orientations (Mavko and Nur, 1975, 1979) (Figure 7). Equations of poroelasticity used in the preceding section require the existence of an RVE, which must be much larger than a typical (or the largest) pore size. Therefore,

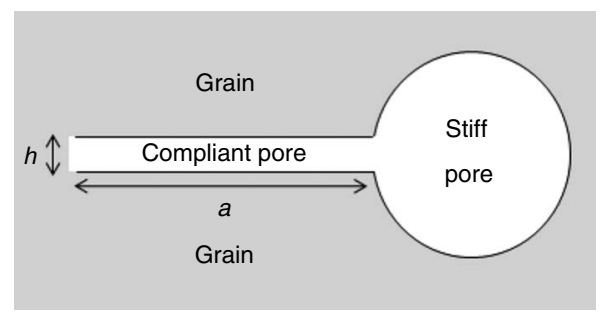

Figure 7. Diagram of squirt WIFF in a granular rock (after Murphy et al., 1986). The soft, or compliant, pore forms a disc-shaped gap between two adjacent grains, and its edge opens into a toroidal stiff pore. When the system is compressed by the passing wave, the compliant pore is compressed to a much greater extent than the stiff one, resulting in the squirt flow from the compliant pore into the stiff pore. 
these equations can only be used to describe processes occurring on scales much larger than pore size. Local or squirt flow occurs on the pore scale and therefore cannot be described by poroelasticity equations (for workarounds, see Pride et al. [2004]).

Most theoretical models of squirt-flow attenuation are based on analyzing aspect-ratio distributions (O'Connell and Budiansky, 1977; Mavko and Nur, 1979; Palmer and Traviolia, 1980); a comprehensive review of these earlier studies is given by Jones (1986). An alternative approach is based on recognizing that the pore space of many rocks has a binary structure (Walsh, 1965; Mavko and Jizba, 1991; Shapiro, 2003): relatively stiff pores, which form most of the pore space, and relatively compliant (or soft) pores, which are responsible for the pressure dependency of the elastic moduli (Murphy et al., 1986; Dvorkin et al., 1995; Chapman et al., 2002). In particular, Dvorkin et al. (1995) model the rock as a granular aggregate in which the grains themselves are assumed porous. Intergranular pores are stiff, whereas the intragranular micropores are soft (compliant). Dvorking et al.'s model is reformulated and refined by Pride et al. (2004).

The advantage of the porous grain model over all other squirt models, particularly in the formulation of Pride et al. (2004), is in the fact that the medium can be treated as poroelastic on the subpore scale and thus is amenable to treatment using Biot's equations of poroelasticity with spatially varying coefficients (Pride and Berryman, 2003a, 2003b). This model is also consistent with Mavko-Jizba predictions for the high-frequency limit of elastic moduli. However, the concept of porous grains is somewhat abstract, and interpretation of parameters of this imaginary microporous grain in terms of rock properties is not straightforward. Furthermore, strictly speaking, application of Biot's theory to microporous grain assumes that compliant pores are small compared to the grain size; this may not be the case for real rocks.

An alternative is the approach of Murphy et al. (1986), who consider compliant pores as gaps at contacts between adjacent grains (see also Mayr and Burkhardt, 2006). However, the model of Murphy et al. is inconsistent with the well-established unrelaxed predictions of Mavko and Jizba (1991) in the high-frequency limit; in fact, its high-frequency predictions for the elastic moduli are unrealistically high. This inconsistency stems from the fact that the particular

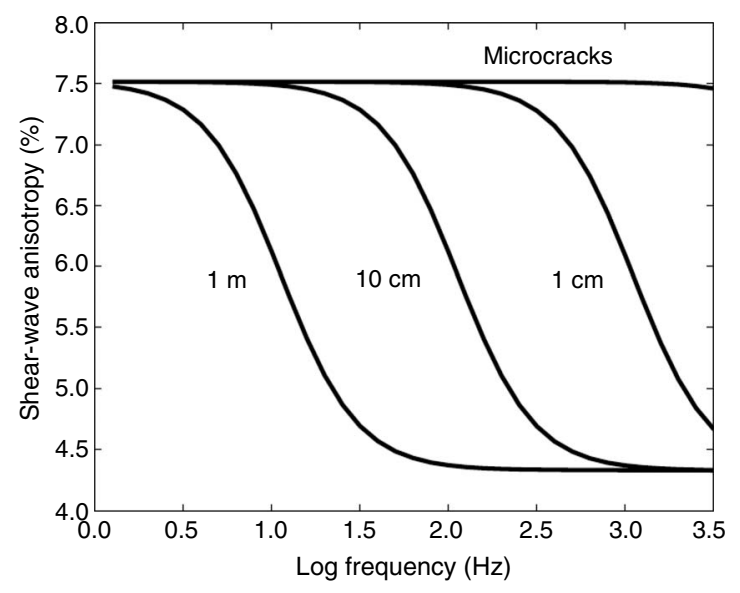

Figure 8. Shear-wave anisotropy as a function of frequency for various fracture sizes $(1 \mathrm{~cm}$ to $1 \mathrm{~m})$, with $10 \%$ equant porosity and a crack density of 0.1 . Propagation is at $70^{\circ}$ to the fracture normal (from Chapman, 2003). formulation of Murphy et al. (1986) is developed within the framework of Hertz-Mindlin grain-contact theory (Digby, 1982; Winkler, 1983), where grains themselves are assumed rigid and the compliance of the rock is caused solely by weak grain contacts. In the highfrequency limit, fluid pressure cannot relax between the intergranular gap and the surrounding (stiff) pore, making its compliance vanishingly small and rock unrealistically stiff.

Recently, Gurevich et al. (2009c) have proposed a new model of squirt-flow attenuation that uses a pressure-relaxation approach of Murphy et al. (1986) in conjunction with the discontinuity tensor formulation of Sayers and Kachanov (1995). The resulting model is consistent with the Gassmann and Mavko-Jizba equations at low and high frequencies, respectively, and with the stress-sensitivity model of Shapiro (2003).

\section{Mesoscopic fractures}

Hudson et al. (1996, 2001) analyze pressure relaxation within a crack through diffusion into the matrix material, the so-called equant porosity model (Thomsen, 1995). Based on general results for the effective properties of cracked solids having aligned or randomly oriented cracks (Hudson, 1981), they derive a dynamic-equivalent stiffness tensor accounting for fluid-pressure diffusion with (matrix) pressure diffusivity $D_{p^{\prime}}=K_{f} \kappa /\left(\phi_{m} \eta\right)$, where $\phi_{m}$ is matrix porosity. The frequency-dependent part of the first-order correction to the stiffness tensor resulting from the presence of a circular crack is of the form (we neglect the tensorial character for brevity)

$$
\tilde{U}(\omega) \propto \frac{1}{1+(1+i) \sqrt{\frac{\omega}{\omega_{c}}}},
$$

with a characteristic frequency

$$
\omega_{c} \approx \frac{\left(\frac{D_{S}^{2}}{D_{p^{\prime}}}\right)}{L^{2}} .
$$

Here, $L$ is the crack radius and $D_{S}=\kappa \mu / \eta$, with $\mu$ the shear modulus of the rock matrix. This results in (P- and $\mathrm{S}$-wave) attenuation asymptotic scalings $Q^{-1} \propto \omega^{1 / 2}$ and $Q^{-1} \propto \omega^{-1 / 2}$ at low and high frequencies, respectively. Hudson et al. (1996) conclude that the equant-porosity mechanism has a significant effect on seismic waves.

Chapman (2003) develops a model for the frequency dependence of the elastic stiffness tensor for an equant-porosity medium in which (1) mesoscale fractures (modeled as an aligned set of oblate spheriods) are embedded with hydraulic connection to the equant pores and (2) microcracks are responsible for squirt flow. The approach is similar to the one used for the squirt-flow model of Chapman et al. (2002). Because the frequency dependence of the full elastic stiffness tensor is considered, estimates of shear-wave attenuation and dispersion resulting from WIFF are obtained. Consequently, the frequency dependence of a shear wave (only caused by waveinduced flow) can be analyzed (see Figure 8).

This model has been extended to account for two mesoscopic fracture sets with different orientations and connectivities (Chapman, 2009). In these models, the fluid mass exchange between frac- 
ture and equant pore is governed by the finite fluid-pressure difference in fracture and equant pore space, resulting in fluid-pressure relaxation of the generic form

$$
\tilde{p}(\omega) \propto p_{0} \frac{\left(\frac{i \omega}{\omega_{c}}\right)}{1+\left(\frac{i \omega}{\omega_{c}}\right)},
$$

with a characteristic frequency

$$
\omega_{c} \approx \frac{D_{S^{\prime}}}{\left(\frac{L a_{c}^{2}}{a_{g}}\right)} .
$$

Here, $L$ is the mesoscale fracture radius, whereas $a_{c}$ denotes the microcrack radius and $a_{g}$ is the grain size. For a small aspect ratio (assumed to be the same for microcracks as well as for mesoscale fractures), the effective diffusivity is given by $D_{S^{\prime}}=\kappa \mu /(\eta[1-\nu])$, with $\nu$ the matrix material Poisson ratio. Assuming a mesoscale fracture radius $L=10 \mathrm{~cm}$ and a microcrack radius $a_{c}=2.5 \mathrm{~mm}$ in a rock volume of water-saturated reservoir sandstone $(\kappa=150 \mathrm{md}$, $\phi=0.15$, average grain size $\left.a_{g}=250 \mu \mathrm{m}\right)$, maximum attenuation is predicted at $f_{c}=\omega_{c} / 2 \pi=220 \mathrm{~Hz}$.

A consequence of fluid-pressure relaxation process 28 is that the attenuations of $\mathrm{P}$ - and $\mathrm{S}$-waves scale at low frequencies such as $Q^{-1}$ $\propto \omega$, in accordance with the models discussed above. However, at high frequencies, the attenuation asymptote scales as

$$
Q^{-1} \propto \frac{1}{\omega} \text {. }
$$

This is different from the asymptotic scaling predicted by Biot poroelasticity-based models with abrupt material property changes (see "3D random continuum models"). In other words, scaling law given by equation 30 implies the diffusion wavelength $\lambda_{p}$ is so small that the transition region between fracture and background medium controls fluid-pressure equilibration.

A more general computational model that can take account of pores and fractures of any size and aspect ratio is proposed by Jakobsen et al. (2003) and Jakobsen (2004) with the T-matrix approximation, commonly used to study effective properties of heterogeneous media (Zeller and Dederich, 1973). In the T-matrix approximation, the effect of voids (pores, fractures) is introduced as a perturbation of the solution for the elastic background medium. Agersborg et al. (2007) show that the model of Chapman (2003) for mesoscale fractures can be obtained as a limit of the more general T-matrix approach. The similarities of both approaches are further investigated by Jakobsen and Chapman (2009).

\section{Models based on viscoelastic rheology and poroviscoelasticity}

Because the WIFF mechanism has the nature of a (fluid-pressure) relaxation phenomenon, it seems logical to model the effect of wave attenuation and dispersion using the phenomenological concept of viscoelastic material behavior (Borcherdt, 2009). This implies the existence of unrelaxed (high-frequency) and relaxed (low-frequency) moduli $M_{\infty}$ and $M_{0}$, respectively. The amount of dispersion is quantified through the modulus defect $\left(M_{\infty}-M_{0}\right) / \sqrt{M_{0} M_{\infty}}$ (Zener, 1948).
One of the most popular and versatile concepts to describe frequency dependence is the so-called standard linear solid (SLS) discussed by Mavko et al. (2009), yielding a complex modulus

$$
\tilde{M}(\omega)=\frac{M_{\infty}\left(M_{0}+i \frac{\omega}{\omega_{c}} \sqrt{M_{\infty} M_{0}}\right)}{M_{\infty}+i \frac{\omega}{\omega_{c}} \sqrt{M_{\infty} M_{0}}} .
$$

It involves a characteristic frequency $\omega_{c}$ (or characteristic relaxation time $\left.\tau_{c}=2 \pi / \omega_{c}\right)$ controlling maximum attenuation. Dvorkin and Mavko (2006) adapt SLS to model attenuation and dispersion of Pand S-waves (and their saturation dependence) in patchy saturated rocks. The critical frequency is then given by $\omega_{c}=D_{p} / a^{2}$, where $a$ is the characteristic patch size and $D_{p}$ is the fluid-pressure diffusivity. Rasolofosaon (2009) phenomenologically models rocks by an assemblage of hysteretic elements and uses SLS to include frequency dependence.

Carcione (1998) modifies Biot's equations to include matrix-fluid interaction mechanisms through viscoelastic relaxation functions. For a finely layered medium, he numerically demonstrates that viscoelastic modeling is not equivalent to porous modeling because of substantial mode conversion from fast wave to slow static mode. However, this effect, caused by WIFF, can be simulated by including an additional relaxation mechanism. Wei and Muraleetharan (2006) develop a linear dynamic model of fully saturated porous media in the spirit of Biot's derivation but include the effect of local (microscopic or mesoscopic) heterogeneities. Local fluid flow is modeled by assuming the pressure difference between solid and fluid phase is proportional to the sum of change in porosity and its time derivative. Complex, frequency-dependent material parameters characterizing the effectively viscoporoelastic medium are derived. The relaxation time depends upon the details of the local structure of porous media that control local fluid-pressure diffusion; the relaxation time is used as a fitting parameter to interpret laboratory data of Paffenholz and Burkhardt (1989). X. Liu et al. (2009) model attenuation from waveinduced flow in double-porosity media using combinations of Zener viscoelastic elements. Picotti et al. (2010) approximate White's (1975) complex bulk modulus using the Zener model and then solve numerically the viscoelastic differential equations.

In the models reported above, the asymptotic behavior of attenuation at high frequencies is $Q^{-1} \propto 1 / \omega$ and therefore can better represent the models based on communicating cavities (previous subsection) but not all models based on Biot's theory of poroelasticity. The viscoelastic models capture the physics of wave-induced flow by choosing suitable combinations of rheological elements. The branching-function approaches discussed above provide an advanced version of the viscoelastic concept in the sense that they are based on asymptotic scaling laws derived from physics-based models. Therefore, the phenomenological character of the viscoelastic models restricts their use for interpretation of measurements in terms of rock properties.

\section{NUMERICAL FORWARD MODELING}

All theoretical models for attenuation and dispersion are based on particular (and simplifying) assumptions such as simple geometric shapes of heterogeneities, low concentration of heterogeneities, or low elastic contrast between the inclusion and embedding medium. It is therefore expedient to develop computer simulation tools that let 
us investigate aspects of the WIFF physics currently unfeasible by theoretical analysis and to verify the validity, accuracy, and range of applicability of theoretical models. Several papers evaluate frequency-dependent attenuation and velocity dispersion in a broad-frequency band (see also Carcione et al., 2010). The most widely used numerical techniques include reflectivity modeling for layered structures and finite-difference and finite-element methods in the time or frequency domain for arbitrary heterogeneous poroelastic structures.

WIFF in 1D heterogeneous structures degenerates to so-called interlayer flow. Attenuation and dispersion from interlayer flow can be modeled using a reflectivity algorithm for poroelastic media (e.g., Schmidt and Tango, 1986). These methods allow numerical models to be constructed for horizontally stratified poroelastic layers by computing the complex plane-wave transmission coefficient along with the associated phase. The coefficients can then be used to calculate the phase velocity $V_{p}$ and attenuation $1 / Q$ in an equivalent homogeneous medium. Gurevich et al. (1997) and Gelinsky et al. (1998) compute $1 / Q$ and $V_{P}$ for randomly layered media and also analyze the interplay between wave-induced flow attenuation and scattering attenuation. Shapiro and Müller (1999) demonstrate that permeability fluctuations result in a significant shift of frequencydependent attenuation and introduce the term seismic permeability to distinguish between an effective permeability controlling wave attenuation from the effective flow permeability (i.e., the harmonic average over all layer permeabilities).

a)

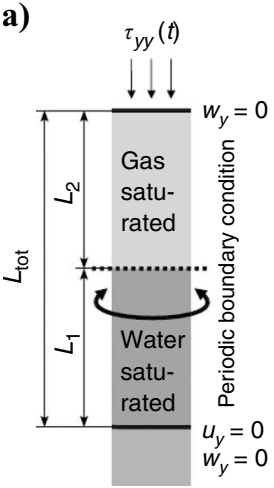

b)
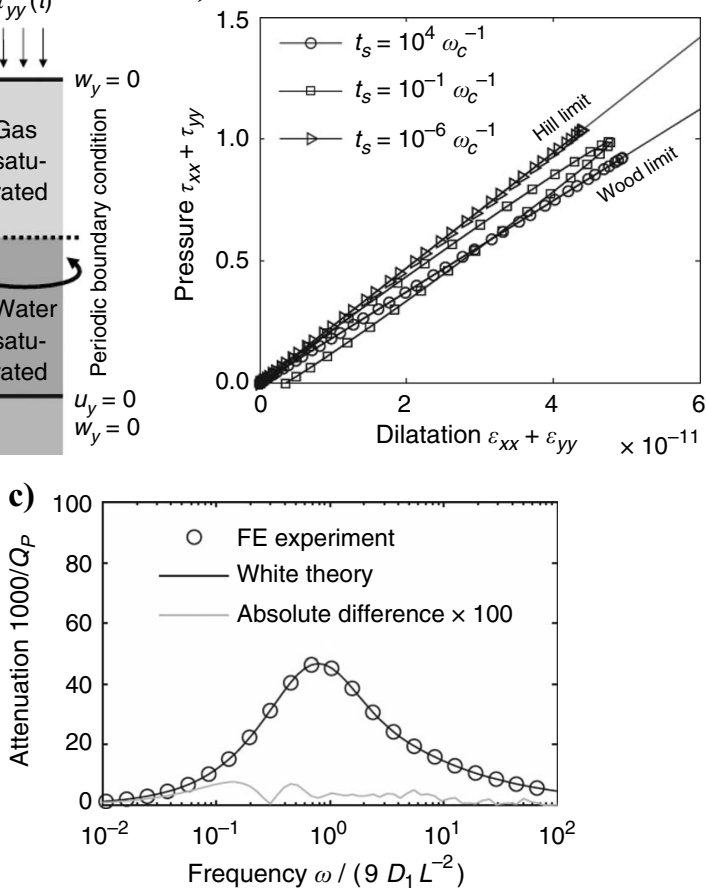

Figure 9. Numerical simulation of wave-induced flow using Biot's equations of poroelasticity. (a) Schematic of the numerical setup of the partial saturation simulation. (b) Hysteresis curves obtained for compressive loading. When the loading is fast, the sample behaves stiffly and the Biot-Gassmann-Hill limit is met. For slow loading, the response is softer and the curves coincide with the Biot-GassmannWood limit. The corresponding frequency-dependent attenuation is shown in (c), where numerical results are compared to the theoretical solution of White (modified after Wenzlau and Müller, 2009).
Modeling well-log data as a sequence of poroelastic layers, Pride et al. (2002) show that interlayer flow attenuation is significant. Lambert et al. (2006) model fractures in a porous medium as a periodic sequence of very thin and compliant layers embedded in a poroelastic background medium and verify the model of Brajanovski et al. (2005). Ren et al. (2009) analyze the normal-incident reflection coefficient as a function of frequency at an interface between an elastic and patchy saturated poroelastic medium and find characteristic reflection-coefficient-frequency relations depending on the impedance contrast. Similarly, Quintal et al. (2009) analyze the magnitude and frequency dependence of the reflection coefficient corresponding to a $\mathrm{P}$-wave reflection from of a thin, patchy saturated poroelastic layer embedded in a homogeneous elastic medium.

The problem of computing attenuation and dispersion in patchy saturated rocks has attracted considerable interest because some exact solutions are available and therefore provide a test for numerical algorithms. Carcione et al. (2003) solve Biot's poroelasticity equations with the finite-difference method to compute attenuation and dispersion for a $2 \mathrm{D}$ periodic arrangement of concentric circles, analogous to the concentric sphere model of White (1975). This modeling strategy has been extended to model a random continuum of fluid distributions (Helle et al., 2003) and general random porous media (J. Liu et al., 2010). Picotti et al. (2007) consider a periodically stratified medium and perform numerical experiments using an iterative domain-decomposition 2D finite-element algorithm for solving Biot's equations of motion in the time domain. The simulated pulses show evidence of the mesoscopic-loss mechanism, and the quality factors estimated with the spectral-ratio and frequency-shift methods are in good agreement with the theoretical values predicted by White's theory.

A fundamental problem in solving Biot's poroelasticity equations on the computer is the numerical stiffness of the system of field equations. This is because the fast, propagating wave modes require a very different spatiotemporal sampling than Biot's slow wave (e.g., Masson et al., 2006). To simulate attenuation and dispersion at seismic frequencies, Masson and Pride (2007) design a quasi-static relaxation simulation using the velocity-stress formulation of the finite-difference method. This approach is generalized by Wenzlau and Müller (2009) using a parallelized version of the velocity-stress finite-difference formulation.

Quasi-static deformation simulations using the finite-element method are an elegant way to compute attenuation and dispersion for arbitrary heterogeneous media (Rubino et al., 2007). In particular, Rubino et al. (2009) design a space-frequency finite-element formulation to compute dynamic-equivalent properties of $2 \mathrm{D}$ randomly inhomogeneous structures. Wenzlau et al. (2010) use time-domain finite-element simulations to obtain attenuation and dispersion for simple-shaped 3D inhomogeneities (see also Figure 9). They also address the problem of attenuation anisotropy (e.g., Zhu and Tsvankin, 2006; Cerveny and Pscenik, 2008) caused by WIFF at aligned heterogeneities.

\section{SUMMARY}

\section{Yet another class of models}

This review has focused on models for attenuation and dispersion where the character of the (mesoscopic) heterogeneities is explicit. That is to say, their characteristic shape, size, and spatial arrangement are captured through suitable geometric parameters, such as fracture thickness, fracture density, and specific surface for discrete 
inclusion models or correlation length for random continua. There exists another category of models for wave attenuation and dispersion in porous solids where the geometric characteristics of heterogeneites are not explicit. In particular, there exists a body of literature on wave propagation in porous media saturated with two or more immiscible fluids. Often these models are constructed by modifying or extending Biot's equations of poroelasticity to include capillary pressure and/or two-phase flow behavior (Berryman et al., 1988; Santos et al., 1990; Lo et al., 2005). Yet another class of models exists that describe acoustic signatures of rocks containing porescale (and larger) gas bubbles through quantification of wave-induced bubble oscillations (Bedford and Stern, 1983; Lopatnikov and Gorbachev, 1987; Commander and Prosperetti, 1989; Smeulders and van Dongen, 1997; Auriault et al., 2002). A comprehensive review of these works is beyond the scope of the present paper. However, some of these approaches result in meaningful interpretations of the aforementioned laboratory measurements, revealing additional facets of the WIFF mechanism not covered by the models reviewed here.

\section{Aspects for future research}

The use of attenuation data in seismic modeling can provide additional information on lithology and fluid content. In principle, the WIFF mechanism provides a link between fluid-transport properties and seismic wave signatures. Pride et al. (2003) discuss the permeability dependence of seismic amplitudes and the potential of estimating flow properties from seismic amplitude analysis. However, to reach this long-standing research goal, more hurdles must be overcome. For example, as Shapiro and Müller (1999) and Müller et al. (2007) point out, in heterogeneous porous media with strong permeability fluctuations, attenuation is not merely controlled by an average flow permeability. Instead, the frequency (and scale) dependence of the flow permeability must be accounted for. And there is abundant evidence that flow permeability can vary by many orders of magnitude even within small rock volumes (Guéguen et al., 1996). More generally, the presence of heterogeneities on various (or many) length scales, as is sometimes reported for petroleum systems, does not permit a simple correspondence between (diffusion) wavelength and heterogeneity scale.

Although the list of theoretical models for wave attenuation and dispersion is long, there are still major research challenges with respect to the applicability, validity range, and experimental verification of most of the models presented in this paper. For example, most models of attenuation and dispersion because of mesoscopic heterogeneities imply that fluid heterogeneities are distributed in a periodic/regular way. In one dimension, this corresponds to periodically alternating layering; in three dimensions, to periodically distributed inclusions of a given shape (usually spheres). All of these models yield similar estimates of attenuation and dispersion. However, experimental studies show that mesoscopic heterogeneities have less idealized distributions and that the distribution itself affects attenuation and dispersion. Further research is required to uncover how to relate the random functions to experimentally significant parameters. The advent of 3D pore-scale imaging of rocks together with numerical simulation tools for evaluation of effective or dynamicequivalent rock properties will certainly contribute to this research goal.

Some of the field observations can be explained consistently with the WIFF mechanism. However, it remains to be seen how accurate- ly the predicted attenuation and dispersion dependencies can be constrained by field observations, given that other fluid- or nonfluid-related attenuation mechanisms may be operative, too. That is why there is significant research demand for controlled laboratory experiments focusing on broad-frequency band (including seismic-frequency band) measurements of wave signatures with additional control of the micro- and mesocopic heterogeneity distribution. To simulate in situ conditions, pressure and temperature controls should also be included in the experimental setup.

\section{ACKNOWLEDGMENTS}

T. Müller acknowledges the financial support of the Deutsche Forschungsgemeinschaft (MU1725/1-3). B. Gurevich and M. Lebedev acknowledge the financial support of the Australian Research Council (Discovery Project DP0771044) and the sponsors of the Curtin Reservoir Geophysics Consortium. The authors thank José Carcione, Yves Guéguen, and Serge Shapiro for helpful reviews.

\section{REFERENCES}

Adam, L., M. L. Batzle, and K. T. Lewallen, 2009, Seismic wave attenuation in carbonates: Journal of Geophysical Research, 114, B06208.

Adelinet, M., J. Fortin, Y. Guéguen, A. Schubnel, and L. Geoffroy, 2010, Frequency and fluid effects on elastic properties of basalt: Experimental investigations: Geophysical Research Letters, 37, L02303.

Agersborg, R., M. Jakobsen, B. O. Ruud, and T. A. Johannsen, 2007, Effects of pore fluid pressure on the seismic response of a fractured carbonate reservoir: Studia Geophysica et Geodaetica, 51, no. 1, 89-118.

Aki, K., and P. G. Richards, 1980, Quantitative seismology: Theory and methods: W. H. Freeman \& Co.

Allard, J. F., 1993, Propagation of sound in porous media: Modelling sound absorbing materials: Chapman \& Hall.

Auriault, J.-L., and C. Boutin, 1994, Deformable porous media with double porosity III: Acoustics: Transport in Porous Media, 14, no. 2, 143-162.

Auriault, J. L., C. Boutin, P. Royer, and D. Schmitt, 2002, Acoustics of a porous medium saturated by a bubbly fluid undergoing phase change: Transport in Porous Media, 46, no. 1, 43-76.

Bacri, J. C., and D. Salin, 1986, Sound-velocity of a sandstone saturated with oil and brine at different concentrations: Geophysical Research Letters, 13, 326-328.

Batzle, M. L., D. H. Han, and R. Hofmann, 2006, Fluid mobility and frequency-dependent seismic velocity — Direct measurements: Geophysics, 71, no. 1, N1-N9.

Bedford, A., and M. Stern, 1983, A model for wave propagation in gassy sediments: Journal of the Acoustical Society of America, 73, 409-417.

Berryman, J. G., 1980a, Long-wavelength propagation in composite elastic media. 1. Spherical inclusions: Journal of the Acoustical Society of America, 68, 1809-1819.

-, 1980b, Long-wavelength propagation in composite elastic media. 2. Ellipsoidal inclusions: Journal of the Acoustical Society of America, 68 1820-1831.

, 2007, Seismic waves in rocks with fluids and fractures: Geophysical Journal International, 171, 954-974.

Berryman, J. G., L. Thigpen, and R. C. Y. Chin, 1988, Bulk elastic wave propagation in partially saturated porous solids: Journal of the Acoustical Society of America, 84, 360-372.

Best, A., and C. McCann, 1995, Seismic attenuation and pore-fluid viscosity in clay-rich reservoir sandstones: Geophysics, 60, 1386-1397.

Biot, M. A., 1956a, Theory of propagation of elastic waves in fluid-saturated porous solid. I. Low-frequency range: Journal of the Acoustical Society of America, 28, 168-178.

$-1956 \mathrm{~b}$, Theory of propagation of elastic waves in a fluid-saturated porous solid. II. Higher frequency range: Journal of the Acoustical Society of America, 28, 179-191.

-1962 , Mechanics of deformation and acoustic propagation in porous media: Journal of Applied Physics, 33, 1482-1498.

Borcherdt, R. D., 2009, Viscoelastic waves in layered media: Cambridge University Press.

Born, W. T., 1941, The attenuation constant of earth materials: Geophysics, 6, 132-148.

Bourbié, T., O. Coussy, and B. Zinszner, 1987, Acoustics of porous media: Editions Technip.

Brajanovski, M., B. Gurevich, and M. Schoenberg, 2005, A model for 
P-wave attenuation and dispersion in a porous medium permeated by aligned fractures: Geophysical Journal International, 163, 372-384.

Brajanovski, M., T. M. Müller, and B. Gurevich, 2006, Characteristic frequencies of seismic attenuation due to wave-induced fluid flow in fractured porous media: Geophysical Journal International, 166, 574-578.

Brajanovski, M., T. M. Müller, and J. O. Parra, 2010, A model for strong attenuation and dispersion of seismic P-waves in a partially saturated fractured reservoir: Science China-Physics, Mechanics and Astronomy, 53, 1383-1387.

Brie, A. F., A. F. Pampuri, F. Marsala, and O. Meazza, 1995, Shear sonic interpretation in gas-bearing sands: Society of Petroleum Engineers Annual Technical Conference and Exhibition, paper 30595-MS.

Brown, R. J. S., and J. Korringa, 1975, On the dependence of the elastic properties of a porous rock on the compressibility of the pore fluid: Geophysics, 40, 608-616.

Cadoret, T., D. Marion, and B. Zinszner B, 1995, Influence of frequency and fluid distribution on elastic wave velocities in partially saturated limestones: Journal of Geophysical Research, 100, 9789-9803.

Cadoret, T., G. Mavko, and B. Zinszner, 1998, Fluid distribution effect on sonic attenuation in partially saturated limestones: Geophysics, 63, $154-160$.

Carcione, J. M., 1998, Viscoelastic effective rheologies for modelling wave propagation in porous media: Geophysical Prospecting, 46, 249-270.

_ 2007, Wave fields in real media: Theory and numerical simulation of wave propagation in anisotropic, anelastic, porous and electromagnetic media, 2nd ed.: Elsevier Scientific Publ. Co., Inc.

Carcione, J. M., H. B. Helle, and N. H. Pham, 2003, White's model for wave propagation in partially saturated rocks, comparison with poroelastic numerical experiments: Geophysics, 67, 1389-1398

Carcione, J. M., C. Morency, and J. E. Santos, 2010, Computational poroelasticity: Geophysics, $\mathbf{7 5}$, this issue.

Carcione, J. M., and S. Picotti, 2006, P-wave seismic attenuation by slow wave diffusion: Effects of inhomogeneous rock properties: Geophysics, 71, no. 3, O1-O8

Carcione, J. M., S. Picotti, D. Gei, and G. Rossi, 2006, Physics and seismic modeling for monitoring $\mathrm{CO}_{2}$ storage: Pure and Applied Geophysics, 163 , 175-207.

Červený, V., and I. Pšenčık, 2008, Quality factor $Q$ in dissipative anisotropic media: Geophysics, 73, no. 4, T63-T75.

Chandler, R. N., and D. L. Johnson, 1981, The equivalence of quasistatic flow in fluid-saturated porous media and Biot's slow wave in the limit of zero frequency: Journal of Applied Physics, 52, 3391-3395.

Chapman, M., 2003, Frequency dependent anisotropy due to mesoscale fractures in the presence of equant porosity: Geophysical Prospecting, 51, 369-379.

- 2009, Modeling the effect of multiple fracture sets of mesoscale fractures in porous rock on frequency-dependent anisotropy: Geophysics, $\mathbf{7 4}$ no. 6, D97-D103.

Chapman, M., S. V. Zatsepin, and S. Crampin, 2002, Derivation of a microstructural poroelastic model: Geophysical Journal International, 151, 427-451.

Ciz, R., and B. Gurevich, 2005, Amplitude of Biot's slow wave scattered by a spherical inclusion in a fluid-saturated poroelastic medium: Geophysical Journal International, 160, 991-1005.

Ciz R., B. Gurevich, and M. Markov, 2006, Seismic attenuation due to waveinduced fluid flow in a porous rock with spherical heterogeneities: Geophysical Journal International, 165, 957-968.

Clark, R. A., P. M. Benson, A. J. Carter, and C. A. Guerrero Moreno, 2009 Anisotropic P-wave attenuation measured from a multi-azimuth surface seismic reflection survey: Geophysical Prospecting, 57, 835-845.

Commander, K. W., and A. Prosperetti, 1989, Linear pressure waves in bubbly fluids: Comparison between theory and experiments: Journal of the Acoustical Society of America, 85, 732-746.

Digby, P. J., 1982, The effective elastic moduli of porous granular rocks: Journal of Applied Mechanics, 48, 803-808.

Domenico, S. N., 1976, Effect of brine-gas mixture on velocity in an unconsolidated sand reservoir: Geophysics, 41, 882-894.

Dutta, N. C., and H. Odé, 1979a, Attenuation and dispersion of compressional waves in fluid-filled porous rocks with partial gas saturation (White model), Part I — Biot theory: Geophysics, 44, 1777-1788.

1979 b, Attenuation and dispersion of compressional waves in fluidfilled porous rocks with partial gas saturation (White model), Part II - Results: Geophysics, 44, 1789-1805

Dutta, N. C., and A. J. Seriff, 1979, On White's model of attenuation in rocks with partial gas saturation: Geophysics, 44, 1806-1812.

Dvorkin, J., and G. Mavko, 2006, Modeling attenuation in reservoir and nonreservoir rock: The Leading Edge, 25, 194-197.

Dvorkin, J., G. Mavko, and A. Nur, 1995, Squirt flow in fully saturated rocks: Geophysics, 60, 97-107.

Endres, A. L., and R. J. Knight, 1997, Incorporating pore geometry and fluid pressure communication into modeling the elastic behavior of porous rocks: Geophysics, 62, 106-117.
Frenkel, J., 1944, On the theory of seismic and seismoelectric phenomena in moist soil: Journal of Physics - USSR, 8, 230-241.

Galvin, R. J., and B. Gurevich, 2007, Scattering of a longitudinal wave by a circular crack in a fluid-saturated porous medium: International Journal of Solids and Structures, 44, 7389-7398.

-2009 , Effective properties of a poroelastic medium containing a distribution of aligned cracks: Journal of Geophysical Research - Solid Earth, 114, B07305.

Gassmann, F., 1951, Über die Elastizität poröser Medien: Vierteljahrsschrift der Naturforschenden Gesellschaft in Zürich, 96, 1-23.

Gelinsky, S., and S. A. Shapiro, 1997, Dynamic-equivalent medium approach for thinly layered saturated sediments: Geophysical Journal International, 128, F1-F4.

Gelinsky, S., S. A. Shapiro, T. M. Müller, and B. Gurevich, 1998, Dynamic poroelasticity of thinly layered structures: International Journal of Solids and Structures, 35, 4739-4752.

Gerner, A., E. H. Saenger, and S. A. Shapiro, 2007, Attenuation of P-waves due to interlayer flow in hydrate-bearing sediments: Journal of Geophysics and Engineering, 4, 394-403.

Gist, G. A., 1994, Interpreting laboratory velocity measurements in partially gas-saturated rocks: Geophysics, 59, 1100-1109.

Gregory, A. R., 1976, Fluid saturation effects on dynamic elastic properties of sedimentary rocks: Geophysics, 41, 895-921.

Guéguen, Y., P. Gavrilenko, and M. LeRavalec, 1996, Scales of rock permeability: Surveys in Geophysics, 17, 245-263.

Guéguen, Y., and V. Palciauskas, 1994, Introduction to the physics of rocks: Princeton University Press.

Gurevich, B., 2003, Elastic properties of saturated porous rocks with aligned fractures: Journal of Applied Geophysics, 54, 203-218.

Gurevich, B., M. Brajanovski, R. Galvin, T. M. Müller, and J. Toms-Stewart, 2009a, P-wave dispersion and attenuation in fractured and porous reservoirs - Poroelasticity approach: Geophysical Prospecting, 57, 225-237.

Gurevich, B., R. J. Galvin, M. Brajanovski, T. M. Müller, and G. Lambert, 2007, Fluid substitution, dispersion and attenuation in fractured and porous reservoirs - Insights from new rock physics models: The Leading Edge, 26, 1162-1168.

Gurevich, B., and S. L. Lopatnikov, 1995, Velocity and attenuation of elastic waves in finely layered porous rocks: Geophysical Journal International, 121, 933-947.

Gurevich, B., D. Makarynska, and M. Pervukhina, 2009b, Ultrasonic moduli for fluid-saturated rocks: Mavko-Jizba relations rederived and generalized: Geophysics, 74, no. 4, N25-N30.

$-2009 \mathrm{c}$, A new squirt-flow model of elastic wave attenuation and dispersion in fluid-saturated rocks: Proceedings of 4th Biot Conference on Poromechanics, 700-705.

Gurevich, B., A. P. Sadovnichaja, S. L. Lopatnikov, and S. A. Shapiro, 1998, Scattering of a compressional wave in a poroelastic medium by an ellipsoidal inclusion: Geophysical Journal International, 133, 91-103.

Gurevich, B., V. B. Zyrianov, and S. L. Lopatnikov, 1997, Seismic attenuation in finely layered porous rocks: Effects of fluid flow and scattering: Geophysics, 62, 319-324.

Helle, H. B., N. H. Pham, and J. M. Carcione, 2003, Velocity and attenuation in partially saturated rock: Poroelastic numerical experiments: Geophysical Prospecting, 51, 551-566.

Hill, R., 1963, Elastic properties of reinforced solids: Some theoretical principles: Journal of Mechanics and Physics of Solids, 11, 357-72.

Hudson, J. A., 1981, Wave speeds and attenuation of elastic waves in material containing cracks: Geophysical Journal of the Royal Astronomical Society, 64, 133-150.

Hudson, J. A., E. Liu, and S. Crampin, 1996, The mechanical properties of materials with interconnected cracks and pores: Geophysical Journal International, 124, 105-112.

Hudson, J. A., T. Pointer, and E. Liu, 2001, Effective-medium theories for fluid-saturated materials with aligned cracks: Geophysical Prospecting, 49, 509-522.

Hughes, D. S., and J. L. Kelly, 1952, Variation of elastic wave velocity with saturation in sandstone: Geophysics, 17, 739-752.

Jakobsen, M., 2004, The interacting inclusion model of wave-induced fluid flow: Geophysical Journal International, 158, 1168-1176.

Jakobsen, M., and M. Chapman, 2009, Unified theory of global flow and squirt flow in cracked porous media: Geophysics, 74 , no. 2 , WA65-WA76.

Jakobsen, M., T. A. Johansen, and C. McCann, 2003, The acoustic signature of fluid flow in complex porous media: Journal of Applied Geophysics, 54, 219-246.

Johnson, D. L., 2001, Theory of frequency dependent acoustics in patchy saturated porous media: Journal of the Acoustical Society of America, 110, 682-694.

Johnson, D. L., J. Koplik, and R. Dashen, 1987, Theory of dynamic permeability and tortuosity in fluid-saturated porous media: Journal of Fluid Mechanics, 176, 379-402.

Johnston, D. H., N. M. Toksöz, and A. Timur, 1979, Attenuation of seismic waves in dry and saturated rocks: II. Mechanisms: Geophysics, 44 
691-711.

Jones, T. D., 1986, Pore fluids and frequency-dependent wave propagation in rocks: Geophysics, 51, 1939-1953.

Karal, F. C., and J. B. Keller, 1964, Elastic, electromagnetic and other waves in random media: Journal of Mathematical Physics, 5, 537-547.

King, M. S., 2009, Recent developments in seismic rock physics: International Journal of Rock Mechanics and Mining Sciences, 46, 1341-1348.

King, M. S., J. R. Marsden, and J. W. Dennis, 2000, Biot dispersion for P- and $\mathrm{S}$-wave velocities in partially and fully saturated sandstones: Geophysical Prospecting, 48, 1075-1089.

Kirstetter, O., P. Corbett, and J. Somerville, 2006, Elasticity/saturation relationships using flow simulation from an outcrop analogue for 4D seismic modelling: Petroleum Geoscience, 12, 205-219.

Klimentos, T., and C. McCann, 1990, Relationships among compressional wave attenuation, porosity, clay content, and permeability in sandstones: Geophysics, 55, 998-1014.

Knight, R., J. Dvorkin, and A. Nur, 1998, Acoustic signatures of partial saturation: Geophysics, 63, 132-138.

Knight, R., and R. Nolen-Hoeksema, 1990, A laboratory study of the dependence of elastic wave propagation of elastic wave velocities on porescale distribution: Geophysical Research Letters, 17, 1529-32.

Konishi, C., H. Azuma, D. Nobuoka, Z. Xue, and J. Watanabe, 2009, Quantitative $\mathrm{CO}_{2}$ saturation estimation from $\mathrm{P}$-wave velocity changes by considering patchy saturation: SEG Summer Research Workshop.

Krzikalla, F., T. M. Müller, B. Hardy, and B. Gurevich, 2006, Seismic wave attenuation and dispersion in patchy-saturated rocks: Numerical experiments: 68th Conference \& Technical Exhibition, EAGE, Extended Abstracts, E038.

Lambert, G., B. Gurevich, and M. Brajanovski, 2006, Attenuation and dispersion of P-waves in porous rocks with planar fractures: Comparison of theory and numerical simulation: Geophysics, 71, no. 3, N41-N45.

Lebedev, M., B. Gurevich, J. Toms, B. Clennell, M. Pervukhina, and T. M. Müller, 2009a, Laboratory observation of velocity-saturation relation transition during water imbibition of porous rock: 4th Biot Conference on Poromechanics, Abstracts, 941-946.

Lebedev, M., J. Toms-Stewart, B. Clennell, V. Shulakova, M. Pervukhina, L. Paterson, T. M. Müller, B. Gurevich, and F. Wenzlau, 2009b, Direct laboratory observation of patchy saturation and its effects on ultrasonic velocities: The Leading Edge, 28, 24-27.

Lee, M. W., and T. S. Collett, 2009, Unique problems associated with seismic analysis of partially gas-saturated unconsolidated sediments: Marine and Petroleum Geology, 26, 775-781.

Lei, X., and Z. Xue, 2009, Ultrasonic velocity and attenuation during $\mathrm{CO}_{2}$ injection into water-saturated porous sandstone: Measurements using difference seismic tomography: Physics of the Earth and Planetary Interiors, 176, 224-234

le Ravalec, M., Y. Guéguen, and T. Chelidze, 1996, Elastic wave velocities in partially saturated rocks: Saturation hysteresis: Journal of Geophysical Research - Solid Earth, 101, 837-844.

Li, X., L. R. Zhong, and L. J. Pyrak-Nolte, 2001, Physics of partially saturated porous media: Residual saturation and seismic-wave propagation: Annual Review of Earth and Planetary Sciences, 29, 419-460.

Liu, E., M. Chapman, I. Varela, X. Li, J. Queen, and H. Lynn, 2007, Velocity and attenuation anisotropy: Implication of seismic fracture characterizations: The Leading Edge, 26, 1170-1174.

Liu, E., J. H. Queen, X. Y. Li, M. S. Maultzsch, H. B. Lynn, and E. M. Chesnokov, 2003, Observation and analysis of frequency-dependent anisotropy from a multicomponent VSP at Bluebell-Altamont field, Utah: Journal of Applied Geophysics, 54, 319-333.

Liu, J., J. Ba, J. W. Ma, and H. Z. Yang, 2010, An analysis of seismic attenuation in random porous media: Science China - Physics, Mechanics \& Astronomy, 53, 628-637.

Liu, X., S. Greenhalgh, and B. Zhou, 2009, Transient solution for poro-viscoacoustic wave propagation in double porosity media and its limitations: Geophysical Journal International, 178, 375-393.

Lo, W. C., G. Sposito, and E. Majer, 2005, Wave propagation through elastic porous media containing two immiscible fluids: Water Resources Research, 41, W02025.

Lopatnikov, S. L., and P. Y. Gorbachev, 1987, Propagation and attenuation of longitudinal waves in a partially gas-saturated porous media: Izvestiya Earth Physics, 23, 683-689.

Lopatnikov, S. L., and B. Gurevich, 1986, Attenuation of elastic waves in a randomly inhomogeneous saturated porous medium: Doklady Earth Science Section, 291, no. 6, 19-22.

Mandelis, A., 2000, Diffusion waves and their uses: Physics Today, 53, no. 8 , 29-34

Markov, M. G., and A. Y. Yumatov, 1988. Acoustic properties of a porous laminated medium: Journal of Applied Mechanics and Technical Physics, 29, 107-111.

Masson, Y. J., and S. R. Pride, 2007, Poroelastic finite difference modeling of seismic attenuation and dispersion due to mesoscopic-scale heterogeneity: Journal of Geophysical Research, 112, B03204.
Masson, Y. J., S. R. Pride, and K. T. Nihei, 2006, Finite difference modelling of Biot's poroelastic equations at seismic frequencies: Journal of Geophysical Research, 111, B10305.

Maultzsch, S., M. Chapman, E. Liu, and X. Y. Li, 2003, Modelling frequency-dependent seismic anisotropy in fluid-saturated rock with aligned fractures: Implication of fracture size estimation from anisotropic measurements: Geophysical Prospecting, 51, 381-392.

- 2007, Modelling and analysis of attenuation anisotropy in multi-azimuth VSP data from the Clair field: Geophysical Prospecting, 55, 627-642.

Mavko, G., and D. Jizba, 1991, Estimating grain-scale fluid effects on velocity dispersion in rocks: Geophysics, 56, 1940-1949.

, 1994, The relation between seismic $\mathrm{P}$ - and $\mathrm{S}$-wave velocity dispersion in saturated rocks: Geophysics, 59, 87-92.

Mavko, G., and T. Mukerji, 1998, Bounds on low frequency seismic velocities in partially saturated rocks: Geophysics, $\mathbf{6 3}, 918-924$.

Mavko, G., T. Mukerji, and J. Dvorkin, 2009, The rock physics handbook, 2nd ed: Cambridge University Press.

Mavko, G., and R. Nolen-Hoeksema, 1994, Estimating seismic velocities at ultrasonic frequencies in partially saturated rocks: Geophysics, 59, 252-258.

Mavko, G., and A. Nur, 1975, Melt squirt in the aesthenosphere: Journal of Geophysical Research, 80, 1444-1448.

, 1979, Wave propagation in partially saturated rocks: Geophysics, 44, $161-178$.

Mayr, S. I., and H. Burkhardt, 2006, Ultrasonic properties of sedimentary rocks: Effect of pressure, saturation, frequency and microcracks: Geophysical Journal International, 164, 246-258.

Milton, G. W., 2002, The theory of composites: Cambridge University Press.

Monsen, K., and S. E. Johnstad, 2005, Improved understanding of velocity saturation relationships using 4D computer-tomography acoustic measurements: Geophysical Prospecting, 53, 173-181.

Müller, T. M., and B. Gurevich, 2004, One-dimensional random patchy saturation model for velocity and attenuation in porous rocks: Geophysics, 69 , $1166-1172$.

,$- 2005 \mathrm{a}$, A first-order statistical smoothing approximation for the coherent wave field in random porous media: Journal of the Acoustical Society of America, 117, 1795-1805.

-2005 b, Wave induced fluid flow in random porous media: Attenuation and dispersion of elastic waves: Journal of the Acoustical Society of America, 117, 2732-2741.

Müller, T. M., B. Gurevich, and S. A. Shapiro, 2008a, Attenuation of seismic waves due to wave-induced flow and scattering in random porous media, in $\mathrm{H}$. Sato, and M. Fehler, eds., Earth heterogeneity and scattering effects on seismic waves: Elsevier Scientific Publ. Co., Inc., 123-166.

Müller, T. M., G. Lambert, and B. Gurevich, 2007, Dynamic permeability of porous rocks and its seismic signatures: Geophysics, 72, no. 5, E149E158.

Müller, T. M., and E. Rothert, 2006, Seismic attenuation due to wave-induced flow: Why Q scales differently in random structures: Geophysical Research Letters, 33, L16305.

Müller, T. M., J. Toms-Stewart, and F. Wenzlau, 2008b, Velocity-saturation relation for partially saturated rocks with fractal pore fluid distribution: Geophysical Research Letters, 26, L09306.

Murphy, W. F., 1982, Effects of partial water saturation on attenuation in Massilon sandstone and Vycor porous glass: Journal of the Acoustical Society of America, 71, 1458-1468.

-1984, Acoustic measures of partial gas saturation in tight sandstones: Journal of Geophysical Research, 89, no. B13, 11549-11559.

Murphy, W. F., J. N. Roberts, D. Yale, and K. W. Winkler, 1984, Centimeter scale heterogeneities and microstratification in sedimentary rocks: Geophysical Research Letters, 11, 697-700.

Murphy, W. F. I., K. W. Winkler, and R. L. Kleinberg, 1986, Acoustic relaxation in sedimentary rocks: Dependence on grain contacts and fluid saturation: Geophysics, 51, 757-766.

Nelson, R. A., 2001, Geological analysis of naturally fractured reservoirs, 2nd ed.: Gulf Publ. Co.

Norris, A. N., 1993, Low-frequency dispersion and attenuation in partially saturated rocks: Journal of the Acoustical Society of America, 94, 359-370.

O'Connell, R. J., and B. Budiansky, 1974, Seismic velocities in dry and saturated cracked solids: Journal of Geophysical Research, 79, 5412-5426. , 1977, Viscoelastic properties of fluid-saturated cracked solids: Journal of Geophysical Research, 82, 5719-5740.

Paffenholz, J., and H. Burkhardt, 1989, Absorption and modulus measurements in the seismic frequency and strain range on partially saturated sedimentary rocks: Journal of Geophysical Research — Solid Earth, 94, 9493 9507.

Palmer, I. D., and M. L. Traviolia, 1980, Attenuation by squirt flow in undersaturated gas sands: Geophysics, 45, 1780-1792.

Parra, J. O., 2000, Poroelastic model to relate seismic wave attenuation and dispersion to permeability anisotropy: Geophysics, 65, 202-210. 
Picotti, S., J. M. Carcione, J. G. Rubino, and J. E. Santos, 2007, P-wave seismic attenuation by slow-wave diffusion: Numerical experiments in partially saturated rocks: Geophysics, 72, no. 4, N11-N21.

Picotti, S., J. M. Carcione, J. G. Rubino, J. E. Santos, and F. Cavallini, 2010, A visoelastic representation of wave attenuation in porous media: Computers and Geosciences, 36, 44-53.

Pride, S. R., 2005, Relationships between seismic and hydrological properties: Hydrogeophysics, 50, 253-290.

Pride, S. R., and J. G. Berryman, 2003a, Linear dynamics of double-porosity and dual-permeability materials. I. Governing equations and acoustic attenuation: Physical Review E, 68, 036603.

- 2003 b, Linear dynamics of double-porosity and dual permeability materials. II. Fluid transport equations: Physical Review E, 68, 036604.

Pride, S. R., J. G. Berryman, and J. M. Harris, 2004, Seismic attenuation due to wave induced flow: Journal of Geophysical Research, 109, no. B1, B01201.

Pride, S. R., J. H. Harris, D. L. Johnson, A. Mateeva, K. T. Nihei, R. L. Nowack, J. W. Rector, H. Spetzler, R. Wu, T. Yamomoto, J. G. Berryman, and M. Fehler, 2003, Acquisition/processing — Permeability dependence of seismic amplitudes: The Leading Edge, 22, 518-525.

Pride, S. R., and Y. J. Masson, 2006, Acoustic attenuation in self-affine porous structures: Physical Review Letters, 97, 184301.

Pride, S. R., F. D. Morgan, and A. F. Gangi, 1993, Drag forces of porous medium acoustics: Physical Review B, 47, 4964-4978.

Pride, S. R., E. Tromeur, and J. G. Berryman, 2002, Biot slow-wave effects in stratified rock: Geophysics, 67, 271-281.

Quintal, B., S. M. Schmalholz, and Y. Y. Podladchikov, 2009, Low-frequency reflections from a thin layer with high attenuation caused by interlayer flow: Geophysics, 74, no. 1, N14-N22.

Rasolofosaon, P. N. J., 2009, Unified phenomenological model for the mechanical behavior of rocks: Geophysics, 74, no. 5, WB107-WB116.

Ren, H., G. Goloshubin, and F. J. Hilterman, 2009, Poroelastic analysis of amplitude-versus-frequency variations: Geophysics, 74, no. 6, N41-N48.

Rice, J. R., and M. P. Cleary, 1976, Some basic stress diffusion solutions for fluid-saturated elastic porous media with compressible constituents: Reviews of Geophysics and Space Physics, 14, 227-241.

Rubino, J. G., C. L. Ravazzoli, and J. E. Santos, 2009, Equivalent viscoelastic solids for heterogeneous fluid-saturated porous rocks: Geophysics, 74, no. $1, \mathrm{~N} 1-\mathrm{N} 13$.

Rubino, J. G., J. E. Santos, S. Picotti, and J. M. Carcione, 2007, Simulation of upscaling effects due to wave-induced fluid flow in Biot media using the finite-element method: Journal of Applied Geophysics, 62, 193-203.

Rudnicki, J. W., 1986, Fluid mass sources and point forces in linear elastic diffusive solids: Mechanics of Materials, 5, 383-393.

Rytov, S. M., Y. A. Kravtsov, and V. I. Tatarskii, 1989, Wave propagation through random media: Springer-Verlag Berlin.

Sams, M. S., J. P. Neep, and M. H. Worthington, 1997, The measurement of velocity dispersion and frequency-dependent intrinsic attenuation in sedimentary rocks: Geophysics, 62, 1456-1464.

Santos, J. E., J. Douglas, J. Corbero, and O. M. Lovera, 1990, A model for wave propagation in a porous medium saturated by a two-phase fluid: Journal of the Acoustical Society of America, 87, 1439-1448.

Sayers, C. M., and M. Kachanov, 1995, Microcrack-induced elastic wave anisotropy of brittle rocks: Journal of Geophysical Research, B100, 4149-4156.

Schmidt, H., and G. Tango, 1986, Efficient global matrix approach to the computation of synthetic seismograms: Geophysical Journal of the Royal Astronomical Society, 84, 331-359.

Schoenberg, M., 1980, Elastic wave behavior across linear slip interfaces: Journal of the Acoustical Society of America, 68, 1516-1521.

Schoenberg, M., and C. M. Sayers, 1995, Seismic anisotropy of fractured rock: Geophysics, 60, 204-211.

Sengupta, M., G. Mavko, and T. Mukerji, 2003, Quantifying subresolution saturation scales from time-lapse seismic data: A reservoir monitoring case study: Geophysics, 68, 803-814.

Shapiro, S. A., 2003, Elastic piezosensitivity of porous and fractured rocks: Geophysics, 68, 482-486.

Shapiro, S. A., and T. M. Müller, 1999, Seismic signatures of permeability in heterogeneous porous media: Geophysics, 64, 99-103.

Shapiro, S. A., E. Rothert, V. Rath, and J. Rindschwentner, 2002, Characterization of fluid transport properties of reservoirs using induced microseismicity: Geophysics, 67, 212-220.

Smeulders, D. M. J., and M. E. H. van Dongen, 1997, Wave propagation in porous media containing a dilute gas-liquid mixture: Theory and experiments: Journal of Fluid Mechanics, 343, 351-373.

Smith, T. M., C. H. Sondergeld, and C. S. Rai, 2003, Gassmann fluid substitutions: A tutorial: Geophysics, 68, 430-440.

Spencer, J. W., 1981, Stress relaxations at low frequencies in fluid-saturated rocks: Attenuation and modulus dispersion: Journal of Geophysical Research, 86, 1803-1812.

Taylor, S. R., and R. J. Knight, 2003, An inclusion-based model of elastic wave velocities incorporating patch-scale fluid pressure relaxation: Geophysics, 68, 1503-1509.

Thomsen, L., 1995, Elastic anisotropy due to aligned cracks in porous rock: Geophysical Prospecting, 43, 805-829.

Toksöz M. N., D. H. Johnston, and A. Timur, 1979, Attenuation of seismic waves in dry and saturated rocks, Part 1: Laboratory measurements: Geophysics, 44, 681-690.

Toms, J., 2008, Effect of fluid distribution on compressional wave propagation in partially saturated rocks: Ph.D. dissertation, Curtin University of Technology.

Toms, J., T. M. Müller, R. Ciz, and B. Gurevich, 2006a, Comparative review of theoretical models for elastic wave attenuation and dispersion in partially saturated rocks: Soil Dynamics and Earthquake Engineering, 26, $548-565$.

Toms, J., T. M. Müller, and B. Gurevich, 2007, Seismic attenuation in porous rocks with random patchy saturation: Geophysical Prospecting, 55, 671-678.

Toms, J., T. M. Müller, B. Gurevich, D. L. Johnson, 2006b, Attenuation and dispersion in partially saturated rock: Random vs periodic models: 68th Conference \& Technical Exhibition, EAGE, Extendend Abstracts, E039.

Toms-Stewart, J., T. M. Müller, B. Gurevich, and L. Paterson, 2009, Statistical characterization of gas-patch distributions in partially saturated rocks: Geophysics, 74, no. 2, WA51-WA64

Tserkovnyak, Y., and D. L. Johnson, 2001, Can one hear the shape of a saturation patch: Geophysical Research Letters, 29, 1108-1112.

, 2003, Capillary forces in the acoustics of patchy-saturated porous media: Journal of the Acoustical Society of America, 114, 2596-2606.

Walsh, J. B., 1965, The effect of cracks on the compressibility of rock: Journal of Geophysical Research, 70, 381-389.

, 1995, Seismic attenuation in partially saturated rock: Journal of Geophysical Research - Solid Earth, 100, 15407-15424.

Wang, Z., 2001, Fundamentals of seismic rock physics: Geophysics, 66, 398-412.

Waterman, P. C., and R. Truell, 1961, Multiple scattering of waves: Journal of Mathematical Physics, 2, 512-537.

Wei, C. F., and K. K. Muraleetharan, 2006, Acoustical characterization of fluid- saturated porous media with local heterogeneities: Theory and application: International Journal of Solids and Structures, 43, 982-1008.

Wenzlau, F., J. B. Altmann, and T. M. Müller, 2010, Anisotropic dispersion and attenuation due to wave-induced fluid flow: Quasi-static finite-element modeling in poroelastic solids: Journal of Geophysical Research, 115, B02074.

Wenzlau, F., and T. M. Müller, 2009, Finite-difference modeling of wave propagation and diffusion in poroelastic media: Geophysics, 74, no. 4, T55-T66.

White, J. E., 1975, Computed seismic speeds and attenuation in rocks with partial gas saturation: Geophysics, 40, 224-232.

- 1986, Underground sound: Applications of seismic waves: Elsevier Scientific Publ. Co., Inc.

White, J. E., N. G. Mikhaylova, and F. M. Lyakhovitskiy, 1976, Low-frequency seismic waves in fluid-saturated layered rocks: Physics of the Solid Earth, 11, 654-659.

Winkler, K., 1983, Contact stiffness in granular porous materials: Comparison between theory and experiment: Geophysical Research Letters, 10, 1073-1076.

- 1985, Dispersion analysis of velocity and attenuation in Berea sandstone: Journal of Geophysical Research, 90, 6793-6800.

Winkler, K., and A. Nur, 1982, Seismic attenuation - Effects of pore fluids and frictional sliding: Geophysics, 47, 1-15.

Wood, A. B., 1941, A textbook of sound: Bell.

Wulff, A.-M., and H. Burkhardt, 1997, Mechanisms affecting ultrasonic wave propagation in fluid-containing sandstones under high hydrostatic pressure: Journal of Geophysical Research, 102B, 3043-3050.

Wyllie, M. R. J., A. R. Gregory, and L. W. Gardner, 1956, Elastic wave velocities in heterogeneous and porous media: Geophysics, 21, 41-70.

Yin, C. S., M. L. Batzle, and B. J. Smith, 1992, Effects of partial liquid gas saturation on extensional wave attenuation in Berea sandstone: Geophysical Research Letters, 19, 1399-1402.

Zeller, R., and P. H. Dederich, 1973, Elastic constants of polycrystals: Physica Status Solidi B — Basic Research, 55, 831-842.

Zener, C., 1948, Elasticity and anelasticity of metals: University of Chicago Press.

Zhu, Y., and I. Tsvankin, 2006, Plane-wave propagation in attenuative transversely isotropic media: Geophysics, 71, no. 2, T17-T30. 